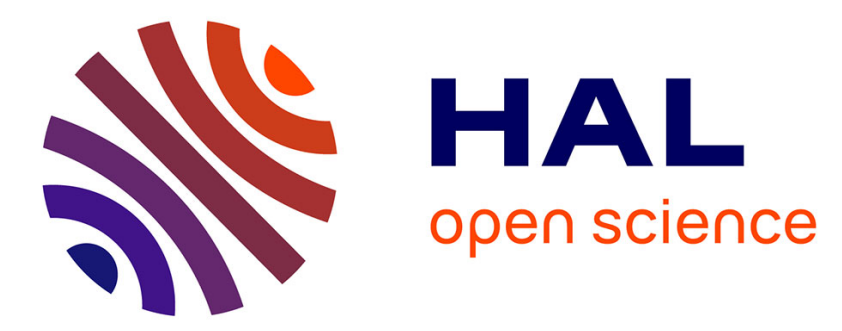

\title{
Les choix de portefeuille des épargnants sur le cycle boursier et le cycle de vie
}

\author{
Alexis Direr, Eric Yayi
}

\section{To cite this version:}

Alexis Direr, Eric Yayi. Les choix de portefeuille des épargnants sur le cycle boursier et le cycle de vie. 2013. halshs-00827239

\section{HAL Id: halshs-00827239 \\ https://shs.hal.science/halshs-00827239}

Preprint submitted on 29 May 2013

HAL is a multi-disciplinary open access archive for the deposit and dissemination of scientific research documents, whether they are published or not. The documents may come from teaching and research institutions in France or abroad, or from public or private research centers.
L'archive ouverte pluridisciplinaire HAL, est destinée au dépôt et à la diffusion de documents scientifiques de niveau recherche, publiés ou non, émanant des établissements d'enseignement et de recherche français ou étrangers, des laboratoires publics ou privés. 


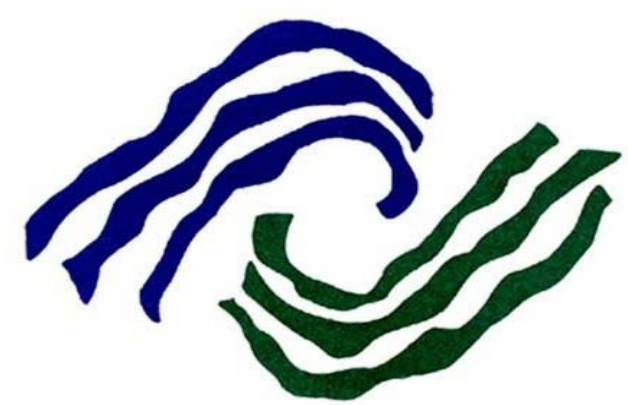

Laboratoire d'Economie d'Orléans

\title{
Document de Recherche
}

$n^{\circ}$ 2013-02

\section{« Les choix de portefeuille des épargnants sur le cycle boursier et le cycle de vie "}

\author{
Alexis DIRER \\ Eric YAYI
}

Laboratoire d'Economie d'Orléans - UMR CNRS 7322 Faculté de Droit, d'Economie et de Gestion, Rue de Blois, B.P. 26739 - 45067 Orléans Cedex 2 - France

Tél : 33 (0)2 38417037 - $33(0) 238494504$ - Fax : 33 (0)2 38417380

E-mail : leo@univ-orleans.fr - http://www.univ-orleans.fr/leo/ 


\title{
Les choix de portefeuille des épargnants sur le cycle boursier et le cycle de vie ${ }^{1}$
}

\author{
A. Direr ${ }^{2}$ \\ Laboratoire d'Economie d'Orléans \\ (LEO - UMR 7322 CNRS) \\ E. Yayi \\ Laboratoire d'Economie d'Orléans \\ (LEO - UMR 7322 CNRS)
}

Janvier 2013

\footnotetext{
1 Nous remercions la société d’assurance qui a aimablement mis à notre disposition les données de cette étude, ainsi que ses collaborateurs et collaboratrices qui nous ont apporté assistance et conseils pour la construction et l'exploitation de la base. Nous remercions également Christophe Hurlin et Nicolas Debarsy pour leurs conseils sur la partie économétrique de l'article. Nous sommes cependant seuls responsables des lacunes qui subsistent.

Laboratoire d'Economie d'Orléans (LEO - UMR 7322 CNRS) et Paris School of Economics. Adresse : Rue de Blois - BP 26739, 45067 Orléans Cedex 2 France. Courriel : direr@ens.fr.
} 


\section{Résumé}

Les épargnants détenant des titres financiers risqués ont-ils tendance à investir à contretemps sur les marchés boursiers, achetant au sommet et vendant dans les creux ? Réduisent-ils leur exposition au risque avec l’âge et en particulier à l'approche de la retraite ? Nous répondons à ces deux questions à l'aide des données d'un grand assureur français répertoriant les souscriptions de contrats Madelin entre 2002 et 2009. Les souscripteurs peuvent placer leur épargne dans deux types de support : un fonds en euros composé essentiellement de titres monétaires quasi sans risque, et des fonds en unités de compte représentant des parts d’OPCVM investies en titres dont le rendement est risqué.

Nous montrons que la part du capital investie en unités de compte est sensible à la conjoncture boursière, mais essentiellement à la date de souscription du contrat. Une fois la part initiale sélectionnée, une forte inertie des choix de portefeuille est observée puisque les épargnants ne reviennent que très rarement sur la décision prise à l'ouverture. Nous constatons une forte procyclicité des choix d'investissement qui s'explique par une extrapolation de la performance boursière récente. Les nouveaux souscripteurs achètent des actifs risqués lorsque la Bourse monte et cessent d'en acheter quand elle descend. Cela les conduit à détenir une part d'actifs risqués minimum en 2004, au début d'une phase de hausse de quatre ans et une part maximum en 2008 au début de la chute boursière liée à la crise financière.

Nous trouvons également que la part risquée décline de façon régulière avec l'âge une fois tenus compte des effets temps et en excluant les effets génération. Le profil par âge décline également dans la configuration inverse (prise en compte des effets génération et exclusion des effets temps) mais la baisse est moins accentuée. Elle est en effet le produit de deux phénomènes. D’une part le nombre d'épargnants investissant dans des actifs risqués tend à s'accroître avec l'âge. D'autre part, conditionnellement à investir, la part risquée diminue avec l'âge. Après une discussion de la plausibilité des différents effets, nous estimons une probabilité de détention d'unités de compte qui croît avec l'âge d'environ 12 points de pourcentage entre 40 et 60 ans, et une part investie en unités de compte conditionnellement à détenir une part positive qui décroît avec l’âge d'environ 6 points de pourcentage entre 40 et 60 ans. La décroissance est trop faible pour amener la part investie à zéro à l’approche de la retraite.

Mots clés : épargne, vieillissement, actifs risqués, choix de portefeuille.

JEL : D91, G11, J14. 


\begin{abstract}
Do investors holding risky financial securities tend to invest in the stock market, buying at the top and selling at the bottom ? Do they reduce their risk exposure with age and especially when approaching retirement? We answer these questions using data on Madelin contracts from a large French insurer over the period 2002 to 2009. Subscribers can invest their savings in two types of investment vehicles: a euro fund composed primarily of money market securities with almost no risk, and unit-linked funds representing shares of money market funds invested in risky securities.

We show that the share of capital invested in unit-linked funds is sensitive to market conditions, but mainly at the date of subscription. Once the initial share has been selected, inertia of portfolio choice is observed as investors rarely revise their position subsequently. We observe a steep procyclicality of investment choices which can be explained by an extrapolation of recent market performance. New subscribers buy risky assets when the stock market rises and stop buying them when it drops. This leads them to hold a minimum share of risky assets in 2004, the beginning of a 4-year rising phase and a maximum share in 2008 at the beginning of the market fall market due to the financial crisis.
\end{abstract}

We also find that the risky share declines steadily with age once time effects are controlled for and cohort effects are excluded. The age profile also declines in the reverse configuration (taking into account cohort effects and excluding time effects) but the decline is less pronounced. It is in fact the product of two phenomena. On one hand the number of investors investing in risky assets tends to increase with age. On the other hand, conditional on investing in risky shares, equity share tends to decrease with age. After a discussion of the plausibility of the different effects, we estimate a probability of unit-linked detention which increases by about 12 percentage points with age between ages 40 and 60, and a conditional equity share which decreases by about 6 percentage points with age between 40 and 60 years. The decrease is too small to bring the invested share to zero when approaching retirement.

Keywords: savings, life cycle household finance, risky assets, portfolio selection.

JEL: D91, G11, J14. 


\section{Introduction}

Peu d'études empiriques analysent les choix de placement financier des épargnants et leur évolution au cours du temps. L’accès aux marchés financiers s'est pourtant élargi depuis une vingtaine d'années et de nombreux épargnants gèrent aujourd'hui un portefeuille de titres. En France, la détention d'actions et d'OPCVM par les ménages se monte en 2011 à 900 milliards d'euros, soit 22,5 \% du patrimoine financier (Banque de France). Environ $20 \%$ de la population investit dans des valeurs mobilières (INSEE, 2010) sous la forme de détentions d'actions cotées ou non cotées ou à travers la souscription de placements collectifs.

Les vingt dernières années ont aussi été le témoin d'un développement spectaculaire des contrats d'assurance vie (17 millions de détenteurs en 2010 pour un encours de 1300 milliards d'euros) et dans une moindre mesure des contrats d'épargne retraite comme les contrats Madelin pour les professions indépendantes (1,1 millions d'adhérents en 2010) et des Plan d'Epargne Retraite Populaire (ou PERP) pour les salariés (2,1 millions d’adhérents en 2010). Dans un contexte de financement privé croissant de la retraite, les souscripteurs de contrats d'épargne souhaitant épargner à long-terme sont orientés vers ces contrats combinant un fonds euros faiblement risqués et des unités de compte investis sur les marchés financiers (actions, obligations, titres monétaires, SCPI, etc.). Les contrats d'assurance vie sont ainsi investis pour 16,7 \% en unités de compte en 2010, ce qui représente 220 milliards d’euros. Cette part était de 10 \% pendant la première moitié des années 1990 (Cour des Comptes, 2012).

La gestion d'un portefeuille de titres nécessite des choix complexes produisant des conséquences potentiellement lourdes sur le rendement à long-terme de l'épargne et sur le niveau de vie. Alors qu'une littérature en forte expansion souligne les défauts de rationalité des ménages en matière de choix financiers (par exemple Campbell, 2006) et que des enquêtes suggèrent qu'un nombre insuffisant de ménages sont capables de maîtriser les concepts financiers de base ${ }^{3}$, il paraît important d'étudier comment les ménages gèrent en pratique leur épargne financière.

La présente étude poursuit un tel objectif en se concentrant sur deux dimensions importantes des choix de portefeuille : comment les épargnants modifient la part investie en actifs risqués en fonction du cycle boursier et au cours de leur cycle de vie. Nous étudierons en particulier dans

3 Selon une étude réalisée par le Crédoc pour l'Institut pour l'éducation financière du public (IEFP), en partenariat avec l'Autorité des marchés financiers, 80 \% des personnes sondées (sur un échantillon de 1502 personnes de plus de 18 ans) reconnaissent qu'ils se sentent un peu perdus en matière de placements financiers. 
quelle mesure les investisseurs individuels adoptent une politique procyclique d'investissement en achetant en période haute du cycle et en vendant en période basse. La notion même de hauts et bas boursiers n’a cependant de sens que si les rendements futurs peuvent être partiellement anticipés. Les études empiriques documentent l'absence de marche aléatoire à moyen terme et montrent que la prime de risque des actions suit une dynamique de retour vers la moyenne sur des horizons de 2 à 10 ans (Campbell et Shiller, 1988, Fama et French, 1988, Koijen et Van Nieuwerburgh, 2007). Campbell et Viceira (1999) démontrent qu'il est possible pour des investisseurs averses au risque d'exploiter la prédictibilité des rendements boursiers en adoptant une politique contracyclique d'investissement. Cette politique augmente sensiblement le rendement de l'épargne. A contrario, une politique procyclique d'investissement réduirait significativement les rendements boursiers.

La seconde question que nous abordons est celle de l'évolution de la part risquée avec l’âge. Nous cherchons en particulier à savoir si les épargnants vendent leurs actifs risqués à l'approche de la retraite afin de protéger leur capital. La connaissance du profil d'investissement par âge a des conséquences pour les marchés financiers. Alors qu’un nombre croissant d’épargnants nés du baby boom prendront leur retraite dans les années à venir, il est important de mieux évaluer leur propension à vendre leurs actifs financiers à mesure qu'ils vieillissent.

Il existe en théorie et en pratique au moins deux raisons pour lesquelles les investisseurs devraient réduire la part de leur richesse investie dans des actifs risqués avec l’âge. Le premier argument est lié à la dynamique de retour vers la moyenne des rendements boursiers trouvée empiriquement et précédemment évoquée. Merton (1969) et Samuelson (1969) montrent qu’en présence de rendements financiers non corrélés dans le temps, un investisseur dont les préférences sont isoélastiques choisit d'investir une part constante de sa richesse en actifs risqués quel que soit l'horizon de son placement. A la fois l'espérance et le risque du rendement final de l'épargne augmentent avec l'horizon ${ }^{4}$. Le poids relatif des deux considérations ne change pas quand l'horizon s’allonge d’où le résultat d’invariance de la part investie en actifs risqués. Si les rendements sont corrélés temporellement négativement à moyen terme comme c’est le cas empiriquement, une durée plus longue de placement atténue le risque du rendement final en rendant plus probable la compensation des baisses et des hausses boursières. La part investie en actifs risqués augmente par conséquent avec l'horizon (Samuelson, 1991). Barberis (2000) montre qu'en tenant compte du

\footnotetext{
$4 \quad$ La variable pertinente pour un investisseur souhaitant évaluer le risque d'une stratégie est le rendement final de sa richesse et non le rendement annualisé. Cette dernière mesure sous-estime dramatiquement le risque des actions (Samuelson, 1963).
} 
risque d'estimation associé à la prédiction des rendements futurs, un investisseur rationnel devrait décroître la part investie en actifs risqués à l’approche de la retraite.

Deuxièmement, dans la mesure où les revenus du travail ne sont pas ou peu corrélés avec les revenus risqués des placements financiers, les premiers jouent un rôle tampon et protègent la consommation des aléas financiers en amortissant les pertes financières dans le temps. Ce mécanisme de lissage perd son efficacité quand l’horizon de l'investisseur diminue. La demande d’actifs risqués devrait par conséquent réduire avec l’âge (Bodie, Merton et Samuelson, 1992, Viceira, 2001).

La politique de réduction des risques financiers à l'approche de la retraite rejoint la recommandation des conseillers en patrimoine. Ces derniers tendent à conseiller l'acquisition d'actions dans un compte d'épargne dès lors que la durée de détention est suffisamment longue. Logiquement, le désinvestissement en actions à l'approche de la retraite est également communément recommandé. Ces deux principes de gestion de portefeuille se sont institutionnalisés depuis quelques années. D’une part les banques et compagnies d'assurance proposent dans les contrats d'épargne des conventions de gestion à horizon dans lesquelles la part risquée du capital diminue automatiquement avec la durée restante de détention. D'autre part, certains gouvernements ont passé des lois visant à promouvoir ce type de gestion. C'est le cas des Etats-Unis dont le Pension Protection Act voté en 2006 contraint les entreprises proposant des plans de retraite à leurs salariés à proposer un fonds dont la part risquée décroît avec le temps (target date fund) comme choix par défaut. En France, le Plan d'Epargne Retraite Populaire (PERP) lancé en 2006 institue également comme choix par défaut l'adhésion du souscripteur à une règle de sécurisation progressive des droits à l'approche de la retraite.

Nous disposons pour notre étude des données d'une grande compagnie d'assurance enregistrant les souscriptions de contrats Madelin ouverts entre mars 2002 et avril 2009, soit environ 8000 contrats. La base inclut des informations sur les souscripteurs (leur sexe, situation familiale, nombre d'enfants, année de naissance, département d’habitation, catégorie professionnelle, profil patrimonial et indice de revenu) et les contrats (canal de distribution, mois et année d'ouverture du contrat, mois et année du versement, montants versés). La base renseigne également sur la part du capital accumulé investie en euros, très faiblement risqués, et en unités de compte composées d’OPCVM exposés au risque des marchés financiers. La dimension panel des données nous permet de suivre l'évolution de la part placée en unités de compte de chaque épargnant. 
Nous montrons que la part du capital investie en actifs risqués choisie par l'épargnant est sensible à la conjoncture boursière, mais essentiellement à la date de souscription du contrat. Une fois la part initiale sélectionnée, une forte inertie des choix de portefeuille est observée puisque les épargnants ne reviennent que très rarement sur la décision prise à l'ouverture. Nous constatons une forte procyclicité des choix d'investissement. Les nouveaux souscripteurs achètent des actifs risqués lorsque la Bourse monte et cessent d'en acheter quand elle descend. Cela les conduit à faire de mauvais choix financiers. La part moyenne investie en actifs risqués atteint ainsi un minimum en 2004 avant quatre années de hausse et un maximum en 2008, année où la Bourse s’engage dans une phase de baisse durable. Nous trouvons également que la part risquée décline de façon régulière avec l'âge une fois tenus compte des effets temps et en excluant les effets génération. Le profil par âge décline également dans la configuration inverse (prise en compte des effets génération et exclusion des effets temps) mais la baisse est moins accentuée. Elle est en effet le produit de deux phénomènes. D’une part le nombre d'épargnants investissant dans des actifs risqués tend à s’accroître avec l’âge. D’autre part, conditionnellement à investir, la part risquée diminue avec l’âge.

Alors que plusieurs articles analysent la capacité des gérants de fonds à anticiper les mouvements boursiers (par exemple Jiang, Yao et Yu, 2007), il existe peu d'études se penchant sur les investisseurs individuels. En France, des analyses sur données agrégées suggèrent que les épargnants investiraient en moyenne à contretemps, achetant aux sommets et vendant dans les creux (Pansard, 2005, Cour des Comptes, 2010). Il n’existe pas à notre connaissance d'analyse empirique fondée sur un panel d’épargnants.

La question de la relation entre l'âge et la part de la richesse investie en actifs risqués a été l'objet de plusieurs études sur données américaines. Americk et Zeldes (2004) utilisent des données de panel d'un grand fonds de pension américain et une série d'enquêtes en coupe sur le patrimoine des américains et montrent que les estimations des effets d'âge sont très sensibles au choix de retenir les effets temps ou les effets génération. Une difficulté rencontrée par les études empiriques provient en effet de l'impossibilité de démêler les effets de l'âge, du temps et de la génération en raison d'une relation linéaire entre les trois variables. Après une analyse approfondie des différentes hypothèses, les auteurs concluent à un effet décroissant de l'âge sur la part investie en actifs risqués. Agnew, Balduzzi et Sunden (2003) utilisent un panel de quatre ans de détenteurs de plans d'épargne retraite et obtiennent un résultat similaire en se fondant sur la même hypothèse d'inclusion des effets temps 
et d'exclusion des effets génération. Poterba et Samwick (1997) font les hypothèses inverses (exclusion des effets temps et inclusion des effets génération) et trouvent un profil par âge croissant.

En France, les enquêtes patrimoine conduites par l'INSEE permettent d'estimer l'effet âge sur des données en coupe. Arrondel et Masson (2003) sur l'enquête 1998 et INSEE Première (2010) sur l'enquête 2010 trouvent une relation en forme de cloche entre l'âge et la part risquée. El Mekkaoui de Freitas, Lavigne, et Mahieu (2001) exploitent l'enquête patrimoine 1998 et estiment un effet d’âge en contrôlant par un grand nombre de variables sociodémographiques et économiques. Ils montrent que les individus les plus âgés sont plus fréquemment détenteurs d’actifs risqués, et que la part d'actifs risqués qu'ils détiennent est significativement plus importante que pour les autres classes d'âge.

Nos résultats apportent un éclairage complémentaire à ceux des enquêtes patrimoine. Comparé à ces enquêtes, notre échantillon se limite aux professions indépendantes épargnant dans des contrats Madelin. Cette catégorie est en moyenne plus riche que la population générale et dispose de revenus moins stables que ceux des salariés (INSEE Première, 2006). Nous utilisons également un concept plus étroit de la part de la richesse investie en actifs risqués puisque nous ne disposons pas d'information sur la composition de la richesse détenue en dehors du contrat d'épargne étudié. Enfin, notre étude se limite aux détenteurs d'épargne retraite pendant la phase d'accumulation, laquelle s'achève quand l'épargnant prend sa retraite et convertit son plan en rentes viagères ${ }^{5}$. Notre source a cependant plusieurs avantages. Les données utilisées sont de type administratif et ne souffrent pas des biais des données déclaratives, non négligeables en matière d'informations sur le patrimoine. Nous disposons également de données de panel, ce qui nous permet de suivre les épargnants au cours du temps et ainsi de mieux identifier les effets d'âge. Enfin, nous disposons de données couvrant la période 2003-2010 pendant laquelle un cycle boursier complet s'est déroulé. Cette caractéristique de nos données s'avère particulièrement utile pour distinguer les effets d'âge des effets temporels.

Le plan de l'article est le suivant. Les données sont présentées dans une section II. L’évolution de la part investie en actifs risqués sur le cycle boursier est analysée dans la section III et sur le cycle de vie la section IV.

\section{Les données}

$5 \quad$ Voir Coile et Milligan (2009) pour l'étude de la désaccumulation d'actifs risqués aux vieux âges. 
L’étude s'appuie sur les données d'un grand assureur français répertoriant les souscriptions de contrats Madelin. Ces contrats d’épargne retraite, créés en 1994, sont destinés aux professions indépendantes non agricoles. Les versements sont déductibles du bénéfice imposable jusqu’à une certaine limite et le capital est transformé en rentes viagères à la retraite. L'épargne retraite complémentaire est une composante importante du revenu à la retraite des indépendants en raison de la relative faiblesse de leur pension publique. Les versements dans les contrats Madelin ont ainsi totalisé deux milliards d'euros en 2009 pour 834000 contrats en phase d’épargne (sources FFSA), soit un versement moyen par contrat de 2400 euros. La proportion de souscripteurs au sein de la population éligible est de 57 \% en 2009 (sources FFSA), ce qui est élevé si nous la comparons à la population salariée dont environ 8 \% possède un plan d’épargne retraite.

Nous disposons des informations concernant tous les épargnants ayant souscrit un contrat entre mars 2002 et avril 2009 et ne l'ayant pas encore liquidé en janvier 2010, soit environ 8000 contrats. Nous disposons de renseignements sur les souscripteurs (leur sexe, année de naissance, département d'habitation, catégorie professionnelle et deux indicateurs de segmentation marketing liés au revenu et au patrimoine) et les contrats (le canal de distribution du contrat, la date d'ouverture et la date des versements). Pour chaque contrat sont également observés les versements en fréquence mensuelle et la part de l'encours investie en unités de compte (UC) constatée chaque fin d'année civile entre 2002 et 2009, exceptée pour l'année 2006 manquante dans la base. Les souscripteurs ont en effet la possibilité de placer leur épargne dans deux types de support : un fonds en euros composé essentiellement de titres monétaires peu risqués et dont le capital est garanti, et des fonds en unités de compte représentant des parts d’OPCVM investies en Bourse et dont le rendement est risqué. Le tableau 1 indique les rendements annuels des supports proposés dans le cadre du contrat étudié. Nous voyons que le rendement du fonds euro fluctue très peu d'une année sur l'autre, contrairement aux rendements des fonds investis en UC, qualifiés d’actifs risqués dans la suite.

Tableau 1. Rendements historiques des fonds proposés dans le contrat Madelin

\begin{tabular}{|l|c|c|c|c|c|c|c|}
\hline & 2007 & 2008 & 2009 & 2010 & 2011 & $\begin{array}{c}\text { rendement } \\
\text { moyen }\end{array}$ & écart-type \\
\hline fonds euro (en \%) & 4,45 & 4,20 & 3,85 & 3,7 & 3,5 & 3,94 & 0,38 \\
\hline fonds actions & 0,42 & $-37,62$ & 25,06 & 8,84 & $-0,85$ & $-3,30$ & 23,02 \\
\hline fonds diversifiés internationaux & $-0,20$ & $-22,91$ & 16,50 & 8,36 & $-3,88$ & $-1,37$ & 14,84 \\
\hline fonds actions internationales & 2,96 & $-48,69$ & 18,91 & 10,26 & $-9,24$ & $-8,87$ & 26,43 \\
\hline fonds actions Europe & 6,33 & $-45,09$ & 26,81 & 2,49 & $-15,99$ & $-8,61$ & 27,04 \\
\hline fonds zone euro & 9,52 & $-17,71$ & $-19,27$ & 12,27 & 2,29 & $-3,53$ & 14,98 \\
\hline fonds actions énergie & 17,11 & $-54,02$ & 50,05 & $-54,02$ & 17,11 & $-15,33$ & 46,94 \\
\hline $\begin{array}{l}\text { fonds d'obligations indexées } \\
\text { sur l'inflation }\end{array}$ & 3,46 & 4,09 & 6,96 & 1,01 & 0,92 & 3,26 & 2,50 \\
\hline
\end{tabular}

Note : les moyennes indiquées sont géométriques. Les écarts-types sont calculés sur les rendements en pourcentage. 
Dans cet article, l'analyse des choix de portefeuille des épargnants se concentre sur la répartition du capital entre le fonds euro du contrat et les supports en UC. La part moyenne investie dans les supports en UC dans les données est de 35 \%. Le graphique 1 présente la distribution des parts d'UC.

Insérez le graphique 1

Environ 84 \% des épargnants investissent en UC, ce qui est élevé. A titre de comparaison, Direr et Visser (2013) exploitent des données d'assurance vie d'un grand assureur français en 2004 et 2005 et montrent que 42 \% des épargnants investissent dans des UC, soit deux fois moins. La différence provient du fait que les professions indépendantes sont en moyenne plus aisées que les détenteurs d'assurance vie. D'autre part, les contrats Madelin ont un horizon de placement en moyenne plus long ce qui favorise la détention d'actions. Au sein de ceux qui investissent, la part d'UC est distribuée de façon relativement homogène avec toutefois quelques pics. Très peu d'épargnants investissent plus des 2/3 de leur capital en UC.

Les épargnants versent en moyenne 2500 euros par an dans la base, ce qui est très proche des 2400 euros de versements moyens publiés par la FFSA pour l'année 2009. A titre de comparaison, les versements moyens dans les contrats d'épargne retraite en direction des salariés (PERP et assimilés) sont d’environ 1700 euros (Carbonnier, Direr et Slimani Houti, 2013).

\section{L’investissement en actifs risqués en fonction du cycle boursier}

Les décisions de placement financier des épargnants sont-elles influencées par les fluctuations passées des marchés financiers ? Le graphique 2 présente l'évolution moyenne de la part investie en UC entre 2003 et 2010 et superpose l'évolution d’un indice boursier représentatif des évolutions des rendements des marchés d'actions.

Insérez le graphique 2 
Nous observons des mouvements amples de la part investie en UC (échelle de gauche) et une corrélation positive avec l'indice boursier de la zone Euro ${ }^{6}$ (échelle de droite). La part moyenne baisse de $31 \%$ en 2003 à 27 \% en 2004 puis remonte de concert avec la partie haussière du cycle à $38 \%$ en 2008 pour ensuite rebaisser à 35 \%, toujours conjointement avec le retournement boursier. Les épargnants investissent sur les marchés à contretemps : la part investie en actifs risqués est minimum en 2004 au moment où le marché boursier s’apprête à connaître quatre années de hausse ininterrompue. Elle est maximum en 2008, année où la Bourse s’engage dans une phase de baisse durable.

Cette évolution est en partie mécanique si les épargnants n’ajustent pas régulièrement leur portefeuille pour corriger les déformations spontanées provenant des plus ou moins-values apparaissant sur la partie en UC. Americk et Zeldes (2004) et Agnew et al. (2003) montrent sur données américaines que les épargnants ajustent rarement la composition de leur plan d'épargne, ce qui provoque une dérive de la part risquée en fonction des aléas boursiers. En l'absence de mesures correctrices, la part investie en actifs risqués tend à s’accroître en période de hausse boursière et à se réduire en phase de baisse, comme dans le graphique 2.

Il est possible de décomposer l'évolution de la part d’UC en une marge extensive (la variation de la proportion d'épargnants investissant en UC) et une marge intensive (la variation de la part d'UC conditionnellement à investir en UC). Les graphiques 3 et 4 représentent respectivement l'évolution des marges extensives et intensives.

\section{Insérez les graphiques 3 et 4}

Nous observons un profil similaire des deux marges d’ajustement et une évolution un peu plus ample de la marge extensive. La proportion de détenteurs d’UC passe ainsi de $71 \%$ en 2003 à $87 \%$ en 2007, soit un saut de 16 points de pourcentage en l'espace de quatre années. Dans le même temps la part d'UC conditionnellement à investir passe de moins de $38 \%$ à près de $44 \%$. Comme pour la marge totale du graphique 2, les deux marges covarient positivement avec l'indice boursier. Un nombre croissant d'épargnants investissent dans des actifs risqués en phase de hausse boursière et pour des montants supérieurs. Le phénomène inverse se produit en phase de baisse.

\footnotetext{
$6 \quad$ La comparaison avec d'autres indices boursiers que le DJ Eurostoxx 50, comme le CAC 40 ou le DJ Industrial Average ne modifierait pas le caractère procyclique de la part en UC étant donnée la forte corrélation des indices boursiers depuis les années 2000.
} 
Nous avons remarqué qu'il était difficile de départager dans l'évolution de la marge intensive les effets des fluctuations boursières des ajustements explicites de la part investie en UC. Cela ne s’applique pas à la marge extensive, laquelle ne dépend pas des rendements financiers et résulte d’un choix délibéré des épargnants. Les fluctuations prononcées des deux marges restent cependant compatibles avec une forme d'inertie des choix de portefeuille. Il se pourrait en effet que les épargnants choisissent une proportion d'UC à l'ouverture du contrat qu'ils conservent ensuite les années futures faute de les ajuster. Pour vérifier cette possibilité, le graphique 5 décompose la part d’UC en fonction de la date de souscription.

\section{Insérez le graphique 5}

Si la date d'ouverture du contrat influençait peu le choix de la part d'UC sur la vie du contrat, les courbes seraient proches et chacune aurait un profil accentué ressemblant au profil moyen du graphique 2. Contrairement à ce scénario, la translation verticale des courbes est importante. Le profil par année d'ouverture est ensuite relativement plat et même décroissant en tendance. Ces deux caractéristiques révèlent conjointement un effet substantiel de la date d'ouverture sur les choix de la part d’UC. Si nous nous attachons par exemple à la phase de hausse toutes années d'ouverture confondues qui s'étale de 2004 à 2008 dans le graphique 2, la part d’UC augmente de 27 à 38 \%, soit de 11 points. Sur la même période l'évolution de la part d’UC pour les épargnants ayant souscrit en 2002 est négative avec une diminution d'environ 4 points. Elle diminue de près de 2 points pour les souscripteurs de 2003. L'intégralité de la hausse de la part d’UC visible sur le graphique 2 pendant la période de boom boursier est donc attribuable aux choix de part d’UC des nouveaux souscripteurs. Une fois cette part choisie, celle-ci tend ensuite plutôt à diminuer avec l'ancienneté du contrat. Ces conclusions sont confirmées par l'analyse des marges intensives et extensives également décomposées par année de souscription. Le graphique 6 présente la fréquence de détention de parts d’UC par année de souscription.

Insérez le graphique 6

La décision d'investir une partie de son épargne dans des UC est extrêmement dépendante de la date d'ouverture mais une fois cette décision prise, celle-ci n'est pas ou très peu remise en cause lors de la vie du contrat. Cet effet reflète sans aucun doute l'influence des performances boursières passées. L’évolution de la fréquence entre les dates d’ouverture épouse en effet assez fidèlement le cycle boursier. Elle baisse en 2003 puis remonte d'environ 23 points de pourcentage pendant la phase de hausse boursière en 2004 et 2005, se stabilise en 2006 et 2007 pour chuter en 2008 et 2009 
de près de 43 points (de $93 \%$ à $50 \%$ ), deux années de mauvaise performance boursière. La translation des courbes par année d'ouverture explique entièrement le profil en cloche et l'amplitude du graphique 3 des fréquences de détention. Le graphique 7 s’intéresse à l'évolution des parts d’UC conditionnellement à investir décomposée par années de souscription.

Insérez le graphique 7

Là encore, la forme en cloche visible dans le graphique 4 s'explique entièrement par les variations des parts d'UC entre dates d'ouverture du contrat. La hausse de 6 points observée entre 2005 et 2008 sur le graphique 4 se transforme à date d'ouverture donnée en une baisse de 5 à 8 points selon la cohorte de contrats. De même, la forte hétérogénéité des parts d’UC par cohorte d'ouverture observable à la dernière date, avec un écart maximum d'environ 24 points entre la cohorte 2006 et la cohorte 2002, s'explique pour l'essentiel par les variations de parts d'UC observées dès la date d'ouverture.

Pour conclure l'analyse des graphiques 5 à 7, nous constatons que la performance boursière récente agit puissamment sur les décisions financières des épargnants l'année de souscription du contrat mais n’intervient quasiment plus ensuite. Ce résultat est en accord avec Nagel et Malmendier (2011) qui montre que les anticipations de rendement financier des ménages et la décision d’investir en titres risqués sont très sensibles à la conjoncture boursière. Notre analyse indique qu'il suffit de quelques années de mauvais ou bons résultats pour modifier radicalement la participation des nouveaux épargnants aux marchés financiers. ${ }^{7}$ Nous trouvons également une forte inertie des choix financiers au cours de la vie du contrat. Ainsi, si les nouveaux épargnants tendent à acheter des UC au mauvais moment du cycle boursier, ils adoptent ensuite une politique de buy and hold et ne vendent pas leurs UC même quand la Bourse baisse.

\section{L'évolution de la part risquée en fonction de l’âge}

Il est important de comprendre comment les épargnants gèrent leur exposition au risque financier en fonction de leur âge et en particulier à l'approche de la retraite. La vente d'un portefeuille boursier en période de creux boursier peut réduire sensiblement les revenus de l’épargne pendant la retraite.

\footnotetext{
$7 \quad$ La Cour des Comptes (2012) rapporte un phénomène similaire dans les contrats d'assurance vie. Alors que les UC représentaient $10 \%$ des provisions mathématiques pendant la première moitié des années 1990, cette part augmente à partir de 1998 et atteint 21,3 \% en 2000 au sommet du pic boursier, avant de retomber à 16,9 \% en 2002 . La part des UC passe ensuite de 17,3 \% en 2004 à 21,7 \% en 2007 lors du second pic boursier, puis retombe à 16,2 \% en 2008.
} 
Notons qu'une sortie en rentes viagères comme c'est le cas dans les contrats Madelin accentue ce risque. Contrairement à un capital dont les retraits peuvent être lissés ou repoussés dans le temps, le niveau des rentes viagères se calcule sur le capital accumulé au moment de la liquidation du plan. La seule marge (coûteuse) à disposition des travailleurs indépendants est de reculer la date de leur retraite. C'est pourquoi il est recommandé d'étaler le risque de pertes financières en vendant progressivement ses actifs risqués plusieurs années avant la retraite.

L'effet de l'âge sur la part investie en UC peut être évalué de deux manières. Nous pouvons regarder la relation statistique entre les deux variables (soit la dérivée totale de la part d’UC par rapport à l'âge) ou estimer une relation en contrôlant par des variables sociodémographiques et économiques susceptibles d'influencer la part investie en UC à travers l'âge (soit la dérivée partielle). La deuxième méthode estime une relation toutes choses égales par ailleurs et nécessite une procédure économétrique. La première démarche est la plus intéressante dans le cadre de notre étude. Nous souhaitons en effet savoir si les épargnants réduisent leur exposition aux risques financiers avec l'âge indépendamment de la question des facteurs sous-jacents (comme la richesse ou la taille du ménage) qui expliquent cette évolution. C’est pourquoi nous commençons par présenter un certain nombre de propriétés graphiques décrivant la relation totale antre l’âge et la part investie en UC.

\section{1/ Exploration graphique de la relation entre l’âge et la part risquée de l'épargne}

Il existe potentiellement trois déterminants importants de la part de l'épargne investie en UC : l'âge, le temps et la génération. Le temps influence les décisions financières par le biais d’événements macro-financiers affectant tous les épargnants à une date donnée, comme une chute boursière. Les épargnants d’une même génération peuvent également subir des influences communes. Par exemple, le revenu permanent diffère d'une génération à l'autre ou encore ceux ayant débuté leur vie par une période de faibles rendements boursiers et d’inflation élevée comme dans les années 70 peuvent s’éloigner durablement des marchés financiers (Nagel et Malmendier, 2011). Une difficulté statistique réside dans l'impossibilité de séparer simultanément ces trois effets. L’âge, la cohorte de naissance et le temps sont en effet indissolublement liés par l'identité temps = date de naissance + âge. Des hypothèses d'identification de l'effet de l'âge sont donc nécessaires et reviennent en pratique à exclure de l'analyse soit les effets temporels, soit les effets génération. L’utilisation de données de panel par rapport à des données en coupe permet d'estimer des effets temporels ou de séparer les effets âge des effets génération. Mais dans tous les cas des conventions restent 
inévitables. Les choix d'identification des effets reposent in fine sur le jugement, l'analyse des différents effets et leur plausibilité.

Il existe deux approches pour estimer l'effet d’âge, en contrôlant par la cohorte de naissance ou par l'année d’observation. Le graphique 8 présente l'influence de l'âge en coupe transversale. Cela revient à négliger les effets générations et neutraliser les effets temps puisque ces derniers agissent seulement sur la translation des courbes.

Insérez le graphique 8

Les épargnants réduisent régulièrement la part investie en UC à mesure qu'ils vieillissent. Remarquons que chaque courbe relie les parts d’UC de générations différentes, ce qui est une présentation valide de l'information seulement en l'absence d'effets génération. Dans ce cas la part d’UC des générations plus âgées est une estimation correcte de ce que sera la part d’UC des générations plus jeunes dans les années futures. Si toutefois des effets génération existent, ces derniers sont mélangés avec les effets d’âge. Il est ainsi possible que l'effet négatif trouvé s’explique en réalité par le fait que les générations plus âgées investissent une part plus faible de leur épargne en actifs risqués. Nous pouvons alternativement contrôler les effets de génération en lieu et place des effets temps en observant l'évolution de la part risquée au sein de chaque cohorte de naissance, comme dans le graphique 9.

Insérez le graphique 9

La translation vers le bas des courbes indique que les générations plus âgées investissent moins en actifs risqués que celles plus jeunes. D’une part, il est possible que les générations plus âgées, en moyenne moins riches, investissent moins sur les marchés financiers. D’autre part, un moindre niveau d'éducation formelle pourrait être associé à un investissement moins fréquent ou plus limité ${ }^{8}$. La décomposition par dates de naissance a l'avantage de suivre une même génération au cours du temps. Si nous suivons l'effet âge qui découle de cette décomposition, celui-ci est très différent de celui observé dans le graphique 8 qui contrôle les effets temps. L’inconvénient de la représentation par cohorte est le pendant de la représentation par année d'observation du graphique précédent. Les effets temps, s’ils existent, sont mélangés avec les effets d’âge. Or, des effets

\footnotetext{
$8 \quad$ Guiso, Haliassos et Jappelli (2003) montrent que le niveau d'éducation a un effet positif et significatif sur la détention d'actions dans un grand nombre de pays européens, même en contrôlant par l'âge, le revenu ou la richesse. Campbell (2006) trouve un résultat similaire pour les Etats-Unis. De même, le niveau d’alphabétisation financière est un prédicteur important de la participation aux marchés financiers (Maarten, Lusardi et Alessie, 2007).
} 
temporels sont visibles sur le graphique 9. Le profil en forme de S inversé de chaque génération est attribuable à l'effet du cycle boursier déjà commenté dans le graphique $1^{9}$. La section précédente montre que cet effet s'explique par les variations de parts d'UC des nouveaux souscripteurs. Pour voir si un même phénomène se produit ici, décomposons le graphique 9 par année d'ouverture des contrats (graphiques 10a à 10f). Les effets d’âge sont maintenant en moyenne négatifs. La forme en S inversé est donc attribuable à un effet date de souscription. Une fois cet effet contrôlé, la part d’UC diminue régulièrement avec l’âge.

\section{Insérez les graphiques 10}

L'effet négatif de l’âge est obtenu en contrôlant par les effets génération et ceux liés à la date d'ouverture des contrats. Ces derniers peuvent s'interpréter comme une forme d'effet temporel persistante. Nous ne pouvons a priori exclure un effet temporel résiduel qui conduirait les épargnants à réduire tendanciellement la part investie en actifs risqués comme dans le graphique 10. En pratique, il paraît cependant difficile de fournir une interprétation convaincante d'un tel effet. D’une manière générale, on observe sur le moyen terme une réduction des coûts d'accès aux marchés financiers avec le développement des fonds indiciels et la gestion des comptes par internet (Bogana, 2008). Si un effet temporel existait, il serait favorable à la détention d’actifs financiers. La fiscalité des revenus et des plus-values des valeurs mobilières a certes été alourdie au cours des années 2000, mais celle-ci n'a pas affecté les contrats Madelin dont la sortie se fait sous forme de rentes viagères soumises à l'impôt sur le revenu. De plus les conditions de déduction des versements du bénéfice imposable et la taxation des rentes perçues à la sortie du contrat sont identiques pour les parts d’UC et libellées en euros. Enfin, les épargnants considérés dans cette étude ont souscrit un contrat dont les conditions générales en termes de frais et de gestion de l’épargne sont restées fixes tout au long de la vie du contrat.

La discussion des différents effets peut être résumée ainsi. La prise en compte d'effets temps conduit à une relation négative entre l'âge et la part investie en UC mais cette relation peut être confondue avec un effet génération dans lequel les générations les plus âgées détiennent moins d’actifs risqués. Si nous contrôlons par les effets liés à la génération et la date de souscription, la relation entre l'âge et la part d’UC est également décroissante, ce qui conforte le résultat d'un effet négatif de l’âge. Enfin, un effet temporel venant réduire la détention d’actifs risqués au cours du

\footnotetext{
9 L'intérêt de disposer d'un cycle boursier complet est ici évident. Si nous n'avions disposé que d'une phase haussière du cycle boursier, comme par exemple entre 2002 et 2008, nous n'aurions pu trancher entre un effet temporel croissant et un effet âge également croissant. Ce problème d'identification se pose entre autres pour Americks et Zeldes (2004) ou Agnew et al. (2003).
} 
temps confondu avec l'effet d’âge est peu plausible. La section suivante confirme les interprétations graphiques à l’aide de modèles économétriques de détention.

\section{2/ Analyse économétrique}

Nous commençons par analyser la part investie en UC à l'aide d'un modèle Tobit. Le modèle Tobit est un modèle de régression censuré qui permet de tenir compte de la contrainte de positivité de la variable part d’UC. La méthodologie revient à supposer que les épargnants détenant un montant nul d'actifs risqués souhaiteraient en détenir une part négative, mais qu’ils en sont empêchés par la contrainte de positivité. Nous incluons dans les variables explicatives des indicatrices d'âge et différents ensembles de contrôles avec soit des effets cohorte, soit des effets temps :

a) des indicatrices de cohorte (une par année de naissance de l'épargnant), d'année d'ouverture du contrat et d’ancienneté du contrat (nombre d’années écoulées entre l'année d’ouverture et celle du versement).

b) les mêmes indicatrices qu'en a) plus des variables sur les souscripteurs (leur sexe, situation familiale, nombre d'enfants, année de naissance, département d’habitation, catégorie professionnelle, indicateur de profil patrimonial et indicatrice de revenu) et les contrats (le canal de distribution du contrat, le versement annualisé, le nombre annuel de versements et la date d'ouverture). L’annexe A fournit une description détaillée de ces variables.

c) des indicatrices d'années d'observation et d'années d'ouverture.

d) les mêmes indicatrices qu'en c) plus les variables décrites en b) sur les souscripteurs et les contrats.

Les analyses précédentes ayant montré l’importance de tenir compte de l'année au cours de laquelle l'épargnant souscrit le contrat, les quatre ensembles de variables contiennent des indicatrices de date de souscription. Les ensembles a) et b) excluent les effets temps de l'analyse et estiment les effets d’âge en contrôlant par les effets génération sans autres variables de contrôle (ensemble a)), ou avec des contrôles additionnels (ensemble b)). Les ensembles c) et d) adoptent les hypothèses inverses : les effets génération sont exclus et les effets d’âges sont estimés en contrôlant par les effets temps avec ou sans variables explicatives additionnelles. Les résultats détaillés des régressions b) et d) sont placés en annexe. Le graphique 11 présente les effets marginaux de l’âge dans un modèle Tobit estimé avec les ensembles de variables explicatives a) à d). L'annexe B décrit le calcul des effets marginaux de variables discrètes dans le cas d'un Tobit. 
Le profil par âge est décroissant dans les quatre ensembles de contrôles, mais est plus accentué quand les effets temps sont pris en compte et les effets génération exclus que dans la configuration inverse. La baisse est d'environ 7 points de pourcentage tous les 10 ans dans le premier cas et seulement de 1,5 points dans le second cas. Conformément à l'analyse du graphique 10, la prise en compte des dates d'ouverture aboutit à un profil décroissant quand les effets générations sont pris en comptes. L'inclusion de contrôles additionnels ne modifie pas la pente mais seulement le niveau.

Il est possible de décomposer la marge totale de variation entre une marge extensive et une marge intensive, à l'image de ce qui a été fait dans la section précédente. Nous commençons par analyser la marge extensive. Nous régressons la probabilité de détenir une part positive d’UC à l'aide d'un modèle Probit en reprenant les quatre ensembles de contrôles précédemment définis. Le graphique 12 indique les effets marginaux de l'âge sur la probabilité de détenir des unités de compte. L'annexe B présente le mode de calcul des effets marginaux.

\section{Insérez le graphique 12}

L'estimation des effets âge est maintenant très différente selon que les effets temps ou génération sont exclus. Si nous excluons les effets temporels et tenons compte des effets de cohorte, d'année d'ouverture et d'ancienneté, nous observons une probabilité stable de détention autour de 34 \% puis une progression jusqu'à l'âge de 59 ans avec une probabilité de détention d'environ 52 \%, soit un gain de 18 points de pourcentage. L'inclusion de contrôles additionnels ne modifie pas la pente mais seulement le niveau du profil. En revanche, si nous excluons les effets génération, le profil par âge est maintenant décroissant. La probabilité de détention entre 38 et 60 ans passe d'un peu plus de 40 $\%$ à $15 \%$.

\section{Insérez graphique 13}

Le graphique 13 présente les effets marginaux de l'âge sur la part d’UC conditionnellement à investir en UC à l'aide d'un modèle Tobit (voir l'annexe B pour le mode de calcul des effets marginaux). Nous obtenons un profil similaire à celui de la marge totale du graphique 11. Le profil par âge est décroissant à partir de 40 ans pour tous les ensembles de contrôles. La part investie en UC baisse d'environ 3 points de pourcentage tous les 10 ans quand les effets temporels sont exclus 
et 6 points quand les effets génération sont exclus.

En conclusion des graphiques 11 à 13, nous constatons certaines différences selon que les effets génération ou temporels sont exclus. L'effet âge sur la part investie en UC, inconditionnelle dans le graphique 11 ou conditionnelle dans le graphique 13, est négatif quelle que soit la configuration retenue mais la pente est plus forte quand les effets génération sont exclus. La probabilité de détenir des actifs risqués diminue également avec l'âge quand les effets génération sont exclus mais augmente cette fois quand ils sont inclus (graphique 12). Il est difficile de départager ces deux représentations des effets d'âge prima facie. Les générations plus âgées sont moins fréquemment détentrices d'actifs risqués, ce qui peut représenter un effet génération authentique qu'il convient de contrôler (l'effet âge s’affaiblit dans ce cas, voire devient positif dans le Probit), mais ce qui peut aussi révéler un effet âge décroissant. Nous pouvons pousser plus loin l’investigation en nous penchant sur les profils de la part d’UC expliqués par les effets génération, année d'observation et année de souscription.

Insérez les graphiques 14

Les graphiques 14 présentent les effets marginaux sur la part investie en UC des indicatrices de cohorte, d'année d’ouverture, et d’année d’observation dans les régressions Probit estimant la probabilité d'investir en UC et dans les régressions Tobit de la part d’UC conditionnellement à investir en UC. Les effets génération et année de souscription s’interprètent comme suit. Les premiers indiquent un plus grand investissent des générations jeunes que des générations plus âgées pour des raisons possibles liées à des différences de niveau d'éducation ou de richesse évoquées précédemment. La probabilité de détenir des UC gagne environ 15 points de pourcentage entre des générations séparées de 10 ans. Conditionnellement à acheter des UC, la part d’UC est ensuite peu différente d'une génération à l'autre, environ 2 points de pourcentage entre des générations séparées de 10 ans. Les effets année de souscription reproduisent fidèlement le cycle boursier et reflètent l'impact de la performance boursière récente sur les choix d'investissement à l'ouverture du contrat. Les effets temporels sont en revanche plus difficiles à interpréter. Ils ne sont pas reliés au cycle boursier une fois tenus compte des effets de la date d'ouverture et ont un profil qui semble aléatoire. Au-delà des fluctuations de court-terme, la probabilité de détention présente une légère tendance à la baisse entre 2002 et 2009, tendance difficilement rationalisable comme précédemment expliquée. Enfin, la variabilité de la part investie en UC conditionnelle est faible, avec une magnitude maximale d'un point de pourcentage sur l'ensemble de la période. C’est pourquoi nous privilégions 
la configuration dans laquelle les effets temps sont exclus.

Au final, nous en concluons que la probabilité de détention d’UC croît avec l'âge d'environ 12 points de pourcentage entre 40 et 60 ans (graphique 12), et que la part investie en UC conditionnellement à investir décroît avec l’âge d'environ 6 points de pourcentage entre 40 et 60 ans (graphique 13). L’amplitude de la baisse est cependant trop faible pour amener la part investie à zéro à l'approche de la retraite. 


\section{Références}

Agnew J., Balduzzi P. et A. Sunden (2003), « Portfolio choice and trading in a large 401(k) plan » American Economic Review, 93 (1), 193-215.

Ameriks J. et S. Zeldes (2004), " How Do Household Portfolio Shares Vary with Age ? » document de travail.

Arrondel L. et A. Masson (2003), " Stockholding in France » in Stockholding in Europe, éd. par L. Guiso, M. Haliassos et T. Jappelli, Palgrave Macmillan Publishers, pp. 75-109.

Barberis N. (2000), " Investing for the Long-Run when Returns are Predictable » Journal of Finance 55 (1), 225-64.

Bodie, Z., Merton, R. C., et Samuelson, W. F. (1992), « Labor supply flexibility and portfolio choice in a life cycle model” Journal of Economic Dynamics and Control, 16, 427-449.

Bogan V. (2008), " Stock Market Participation and the Internet » Journal of Financial and Quantitative Analysis 43 (1), 191-211.

Campbell J. (2006), « Household Finance » Journal of Finance 61, 1553-1604.

Campbell J. et R. Shiller (1988), "Stock prices, earnings, and expected dividends » Journal of Finance 43, 661-676.

Campbell J. et L. Viceira (1999), « Consumption and Portfolio Decisions When Expected Returns Are Time Varying » Quarterly Journal of Economics 114 (2), 433-95.

Carbonnier C., Direr A. et I. Slimani-Houti (2013), « Do Savers Respond to Tax Incentives? The Case of Retirement Savings », document de travail.

Coile C. et K. Milligan (2009), « How household portfolios evolve after retirement: the effect of ageing and health shocks » Review of Income and Wealth 55 (2), 226-248.

Cour des Comptes (2012), «La politique en faveur de l'assurance vie » Rapport public thématique.

Direr A. et M. Visser (2013), « Portfolio Choice and Financial Advice », Finance, à paraître.

Fama E. et K. French (1988), « Dividend yields and expected stock returns » Journal of Financial Economics 22, 3-27.

Guiso L., Haliassos M., et T. Jappelli (2003), « Stockholding in Europe: Where Do We Stand and Where Do We Go? » Economic Policy, 18, 123-170.

INSEE (2006), « Les indépendants » INSEE Première Nº 1084.

INSEE (2010), « Patrimoine des ménages début 2010 » INSEE Première Nº 1325.

Jiang G., Yao T. et T. Yu (2007), « Do mutual funds time the market? Evidence from portfolio holdings », Journal of Financial Economics 86, 724-758. 
Koijen R. et S. Van Nieuwerburgh (2007), " Market Efficiency and Return Predictability » document de travail.

El Mekkaoui-De Freitas N., Lavigne A. et R. Mahieu (2001), « La détention d'actifs risqués selon l’âge : une étude économétrique », Revue d'Economie Politique, 111, 59-78.

Maarten, V. R., A. Lusardi, et R. Alessie (2007), « Financial Literacy and Stock Market Participation » Michigan Retirement Research Center Research Paper No. 2007-162.

Merton R. (1969), " Lifetime portfolio selection under uncertainty : The continuous time case » Review of Economics and Statistics 51 (3) 247-57.

Nagel S. et U. Malmendier (2011), « Depression babies : Do Macroeconomic Experiences Affect Risk-Taking » Quarterly Journal of economics 126 (1) 373-416.

Pansard F. (2005), "Les tendances du patrimoine financier des ménages français " Revue mensuelle de l’Autorité des Marchés Financiers Nº13.

Poterba J., et A. Samwick (1997), « Household Portfolio Allocation over the Life Cycle » document de travail NBER No. 6185.

Samuelson P. (1963), « Risk and Uncertainty: A Fallacy of Large Numbers », Scientia, 98: 108113.

Samuelson P. (1969), "Lifetime Portfolio Selection by Dynamic Stochastic Programming ", Review of Economic and Statistics, 51, 239-24.

Samuelson P. (1991), « Long-run risk tolerance when equity returns are mean regressing: pseudo paradoxes and vindication of businessman's risk » In Money, macro economics and economic policy: essays in honour of James Tobin. Edité par Brainard W.C, Dorhaus W.D, et Watts H.W pp. 181-200. Eds. Cambridge, MA; London, UK: The MIT Press.

Viceira L. (2001), "Optimal portfolio choice for long-horizon investors with nontradable labor income » Journal of Finance 56 (2), 433-470. 


\section{Annexe A : description des variables explicatives de la base}

Les variables de contrôles utilisées dans les régressions économétriques et nécessitant une définition sont les suivantes :

Segmentation marketing: classification des épargnants sur la base de critère géo-socioprofessionnels.

Revenu : nous disposons pour chaque client d'un indice de revenu produit par le service marketing. Plus l'indice est élevé et plus le revenu estimé du client l'est également. Cet indice permet de construire la variable indicatrice revenu supérieur qui prend la valeur 1 si l’indice est supérieur à un seuil donné.

Profil patrimonial : les clients sont segmentés en différentes catégories patrimoniales selon l'importance du patrimoine investi chez l'assureur et le type de gestion choisi.

Versement annualisé : versements totaux sur l’année civile.

Nombre de versements : nombre de versements par an. Compris entre 1 et 12 .

Canal de distribution : les contrats d'épargne sont souscrits à travers deux canaux de distribution, les agents généraux et les agents spécialisés en assurance des personnes (ASAP). Les deux réseaux sont composés d'entrepreneurs indépendants bénéficiant d'un mandat exclusif avec l'assureur. Les premiers ont pignon sur rue et vendent l'ensemble des produits d'assurance et d'épargne de l'assureur. La plupart d'entre eux font cependant la majeure partie de leur chiffre d'affaires en assurance des biens et des personnes plutôt que sur le segment de l'épargne. Les seconds sont spécialisés dans les domaines de la retraite, l'épargne et les placements financiers. Leurs clients sont généralement des professions libérales, chefs d’entreprises ou cadres supérieurs.

L’ancienneté du contrat : désigne le nombre d’années écoulées entre l'année d'ouverture du contrat et l'année d'observation. 


\section{Annexe B : Calcul des effets marginaux d'une variable discrète dans le cas d'un Tobit et d'un Probit}

Les graphiques 11 à 13 présentent les effets marginaux sur la part investie en UC des indicatrices d'âge. Les formules habituelles des effets marginaux s'appliquent aux variables continues et non aux variables discrètes. Dans le cas d'un Tobit, l'effet marginal (em) associé à la variable indicatrice $\mathrm{Xk}$ pour la part en UC de l'observation $i$ (un individu $j$ pour une année observée $t$ ) est obtenu en mesurant l'impact de la variation totale de $X^{k}$ sur la prédiction de $Y_{i}$ :

$$
\operatorname{em}_{i}\left(X^{k}\right)=\mathrm{E}\left(Y_{i} \mid X_{i}^{k}=1\right)-\mathrm{E}\left(Y_{i} \mid X_{i}^{k}=0\right)
$$

Nous pouvons alors déduire l'effet marginal moyen de l'échantillon en prenant la moyenne des effets marginaux individuels :

$$
\mathrm{em}\left(X^{k}\right)=\frac{1}{N} \sum_{i=1}^{N} \mathrm{em}_{i}\left(X^{k}\right)
$$

Les effets marginaux ainsi calculés sont reportés dans le graphique 11. Nous adoptons la même démarche pour le calcul des effets marginaux conditionnellement à acheter des UC. L'effet marginal individuel emc s'écrit :

$$
\operatorname{emc}_{i}\left(X^{k}\right)=\mathrm{E}\left(Y_{i} \mid Y_{i}>0, X_{i}^{k}=1\right)-\mathrm{E}\left(Y_{i} \mid Y_{i}>0, X_{i}^{k}=0\right)
$$

Les effets marginaux reportés dans le graphique 13 sont les moyennes arithmétiques des effets marginaux individuels. De façon similaire, les effets marginaux individuels dans le Probit s'écrivent :

$$
\operatorname{emt}_{i}\left(X^{k}\right)=\operatorname{Prob}\left(Z_{i}=1 \mid X_{i}^{k}=1\right)-\operatorname{Prob}\left(Z_{i}=1 \mid X_{i}^{k}=0\right)
$$

avec $Z_{i}=1$ si la part en UC associée à l'observation $i$ est strictement positive et $Z_{i}=0$ sinon. Les effets marginaux présentés dans le graphique 12 sont les moyennes des effets individuels calculés sur toutes les observations. 


\section{Annexe C : Régressions Tobit sur les parts investies en unités de compte}

\begin{tabular}{|c|c|c|}
\hline Variables & Effets cohortes exclus & Effets temps exclus \\
\hline \multicolumn{3}{|l|}{ Sexe (référence : homme) } \\
\hline Femme & $-0,0173 * * *(0,0024)$ & $-0,0173^{* * *}(0,0024)$ \\
\hline \multicolumn{3}{|c|}{ Situation familiale (référence : célibataire, divorcé ou veuf) } \\
\hline Concubin ou marié & $-0,0042(0,0027)$ & $0,0039(0,0027)$ \\
\hline Autre & $-0,0395 * * *(0,0041)$ & $-0,0402^{* * *}(0,0041)$ \\
\hline \multicolumn{3}{|l|}{ Enfant (référence : non) } \\
\hline Oui & $0,0042(0,0026)$ & $0,0042(0,0026)$ \\
\hline \multicolumn{3}{|l|}{ CSP (référence : artisans commerçants) } \\
\hline Professions libérales & $0,0216 * * *(0,0036)$ & $0,0218 * * *(0,0036)$ \\
\hline Chefs d'entreprise et cadres supérieurs & $0,0182 * * *(0,0049)$ & $0,0176 * * *(0,005)$ \\
\hline Agriculteurs et autres & $0,0110 * *(0,0054)$ & $0,0106 * *(0,0054)$ \\
\hline \multicolumn{3}{|c|}{ Segmentation marketing (référence : classe moyenne) } \\
\hline Beaux quartiers & $0,0113 * *(0,0047)$ & $0,0116 * *(0,0047)$ \\
\hline Classe moyenne aisée & $-0,01 * *(0,0048)$ & $-0,0102 * *(0,0048)$ \\
\hline Petits logements de ville & $0,0056(0,0046)$ & $0,0055(0,00046)$ \\
\hline Ouvriers touchés par le chômage & $-0,0017(0,0063)$ & $-0,0015(0,0063)$ \\
\hline Retraités & $0,0019(0,0074)$ & $0,0013(0,0075)$ \\
\hline Camp, et zones agricoles & $-0,0046(0,0044)$ & $-0,0045(0,0043)$ \\
\hline Autres & $-0,0054(0,0048)$ & $-0,0059(0,0048)$ \\
\hline \multicolumn{3}{|l|}{ Revenu (référence : revenu inférieur) } \\
\hline Revenu supérieur & $0,0153 * * *(0,0047)$ & $0,0152 * * *(0,0047)$ \\
\hline \multicolumn{3}{|l|}{ 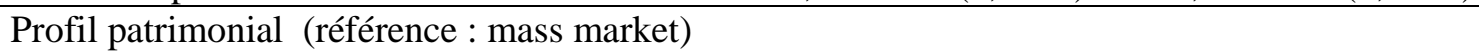 } \\
\hline Affluent sup & $-0,0053(0,0095)$ & $-0,007(0,0095)$ \\
\hline Programme Exclusif & $0,0107 *(0,0054)$ & $0,0097 * * *(0,0054)$ \\
\hline Affluent & $-0,0016(0,0038)$ & $-0,0026 \quad(0,0038)$ \\
\hline Versement annualisé & $-0,0062 * * *(0,001)$ & $-0,0063 * * *(0,001)$ \\
\hline Nombre de versements & $-0,0005 *(0,0003)$ & $-0,0006 * *(0,0003)$ \\
\hline \multicolumn{3}{|c|}{ Canal de distribution (référence : agents généraux) } \\
\hline Agents spécialisés en assurance des personnes & $0,0273 * * *(0,0029)$ & $0,0272 * * *(0,0029)$ \\
\hline \multicolumn{3}{|l|}{ Année d'ouverture (référence : 2002) } \\
\hline 2003 & $-0,0312 * * *(0,0038)$ & $-0,0391 * * *(0,0055)$ \\
\hline 2004 & $0,0723^{* * *}(0,0041)$ & $0,0577 * * *(0,0088)$ \\
\hline 2005 & $0,2028 * * *(0,0044)$ & $0,1819 * * *(0,0126)$ \\
\hline 2006 & $0,2133 * * *(0,004)$ & $0,1816 * * *(0,0162)$ \\
\hline 2007 & $0,2059 * * *(0,0045)$ & $0,1671 * * *(0,0201)$ \\
\hline 2008 & $0,0973 * * *(0,0056)$ & $0,0501 * *(0,0242)$ \\
\hline 2009 & $-0,0968 * * *(0,0137)$ & $-0,1465 * * *(0,0306)$ \\
\hline Indicatrices d'âge & $\overline{\text { oui }}$ & oui \\
\hline Indicatrices d'année d'observation & oui & non \\
\hline Indicatrices de cohorte de naissance & non & oui \\
\hline Indicatrices d'ancienneté du contrat & non & oui \\
\hline Indicatrices de département & oui & oui \\
\hline Nombre d'observations (AIC) & $34188(1940)$ & $34188(1950)$ \\
\hline
\end{tabular}

Echantillon : ensemble des contrats Madelin souscrits entre 2002 et 2009, exceptée l'année 2006, année pour laquelle les parts d'UC sont manquantes. * : significatif au seuil de $10 \%$; ** : significatif au seuil de $5 \%$; *** : significatif au seuil de $1 \%$. Entre parenthèses : écart type. AIC : Critère d'information d'Akaike.

Notes : Le tableau présente les résultats d'un modèle Tobit expliquant la part investie en unités de compte. Les variables explicatives sont décrites dans l'annexe A. La première colonne «Effets cohortes exclus » inclut toutes les variables explicatives dont les indicatrices de temps, exceptés les indicatrices de cohorte de naissance et d'années d'ancienneté du contrat. La deuxième colonne « Effets temps exclus » inclut toutes les variables explicatives dont les indicatrices de cohorte, exceptés les indicatrices de temps. Les effets marginaux des indicatrices d'âge sont reportés dans le graphique 11. 
Graphique 1. Distribution des parts investies en UC.

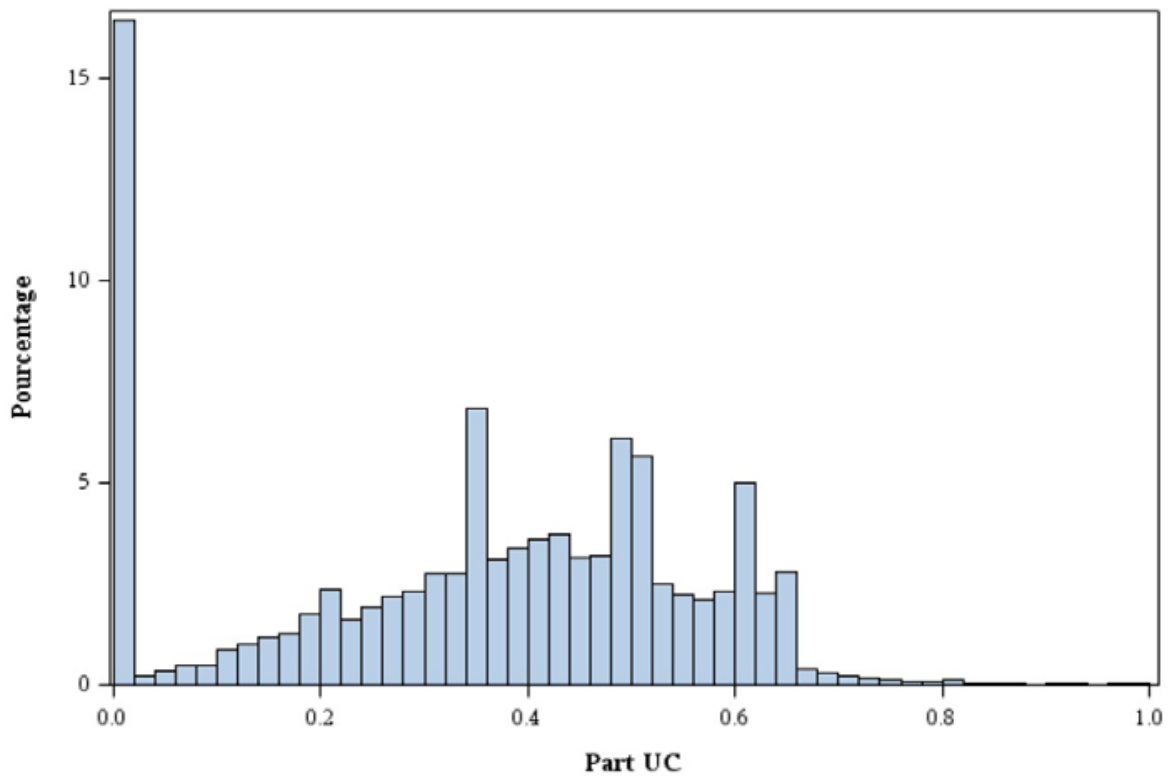

Echantillon : ensemble des contrats Madelin souscrits entre 2002 et 2009, exceptés l'année 2006.

Graphique 2. Evolution de la part investie en unités de compte (échelle de gauche) et indice boursier DJ Eurostoxx 50 (échelle de droite)

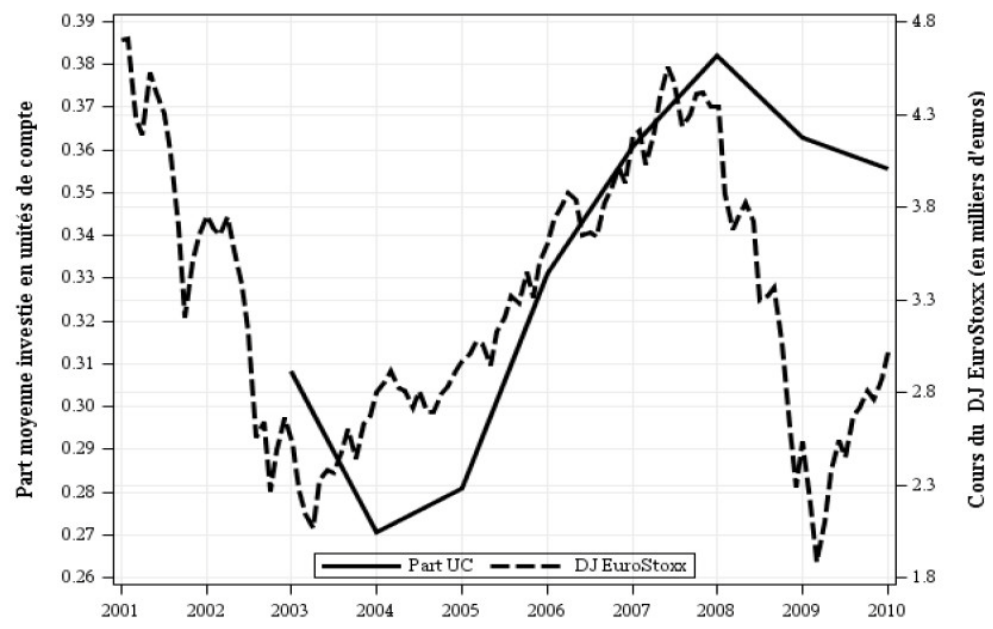

Echantillon : ensemble des contrats Madelin souscrits entre 2002 et 2009, et données mensuelles du Dow Jones EuroStoxx 50 (regroupe 50 sociétés selon leur capitalisation boursière au sein de la zone Euro).

Notes : Les parts d’UC de l'année $n=2002, \ldots, 2009$ sont observées au 31 décembre. Elles sont datées en abscisse le 1er janvier de l'année $n+1$. Les parts investies en UC de l'année 2006 sont manquantes dans la base et sont obtenues par interpolation au niveau contrat. 
Graphique 3. Evolution de la proportion d'épargnants investissant en unités de compte (échelle de gauche) et indice boursier DJ Eurostoxx 50 (échelle de droite)

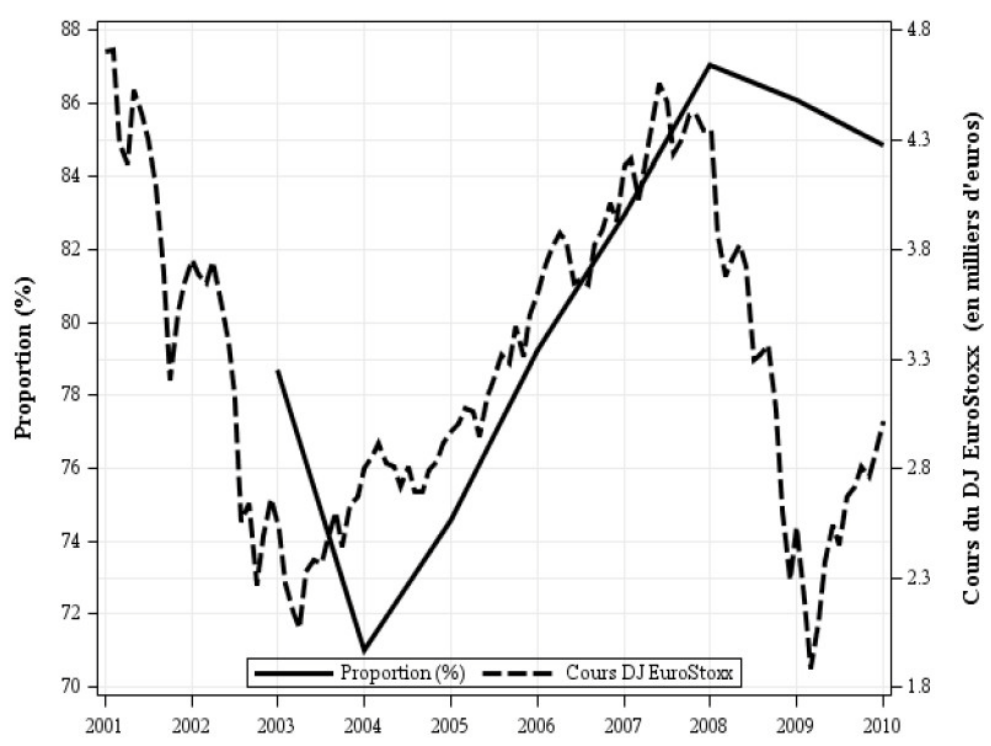

Echantillon : ensemble des contrats Madelin souscrits entre 2002 et 2009, et données mensuelles du Dow Jones EuroStoxx 50.

Notes : Les parts d'UC de l'année $n=2002, \ldots, 2009$ sont observées au 31 décembre. Elles sont datées en abscisse le 1er janvier de l'année $n+1$. Les parts investies en UC de l'année 2006 sont obtenues par interpolation au niveau contrat.

Graphique 4. Evolution de la part d'unités de compte conditionnellement à acheter des unités de compte (échelle de gauche) et indice boursier DJ Eurostoxx 50 (échelle de droite)

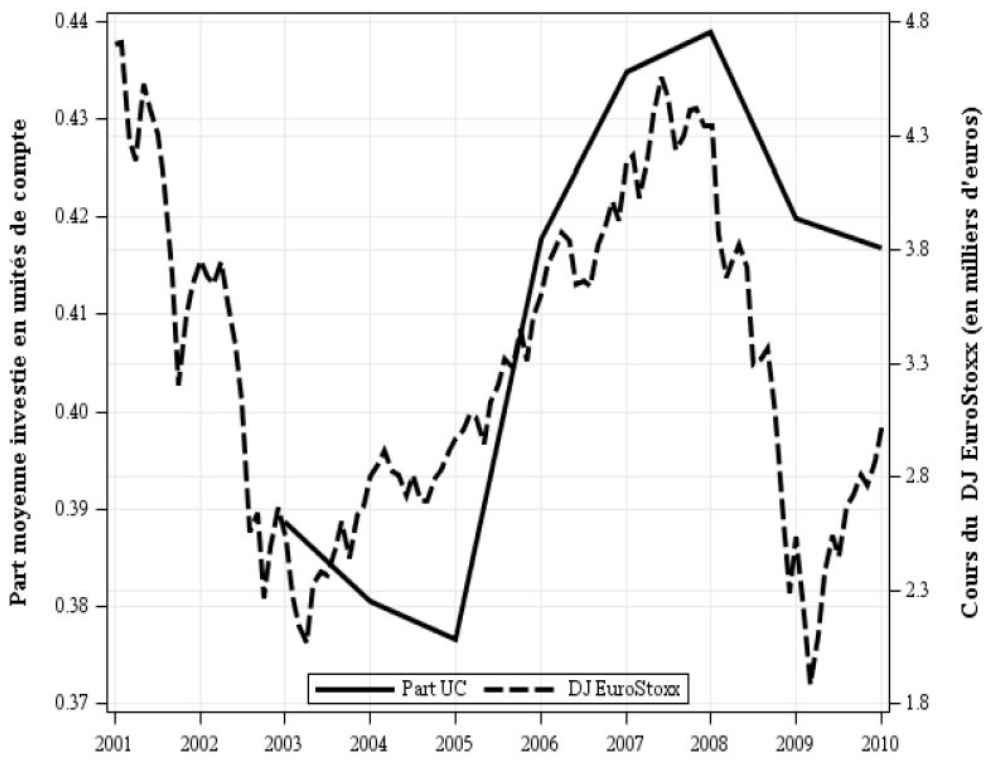

Echantillon : ensemble des contrats Madelin souscrits entre 2002 et 2009, et données mensuelles du Dow Jones EuroStoxx 50.

Notes : Les parts d’UC de l'année $n=2002, \ldots, 2009$ sont observées au 31 décembre. Elles sont datées en abscisse le 1er janvier de l'année $n+1$. Les parts investies en UC de l'année 2006 sont obtenues par interpolation au niveau contrat. 
Graphique 5. Vue par années d’ouverture des parts investies en UC en fonction du temps

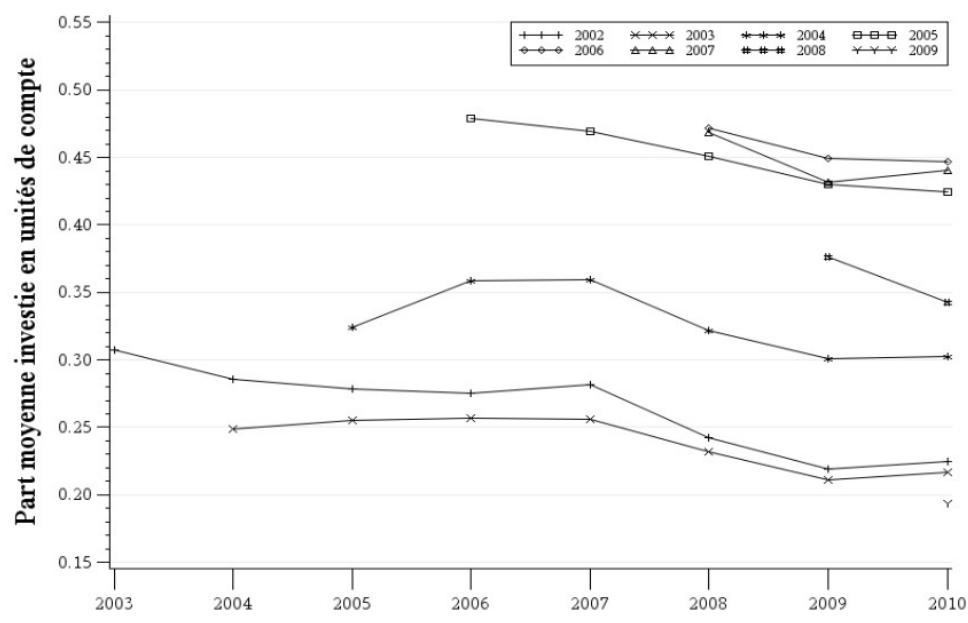

Echantillon : ensemble des contrats Madelin souscrits entre 2002 et 2009. Les parts d'UC de l'année 2006 sont obtenues par interpolation au niveau contrat.

Graphique 6. Evolution des proportions d'épargnants ayant investi en unités de compte, décomposée par années d'ouverture du contrat

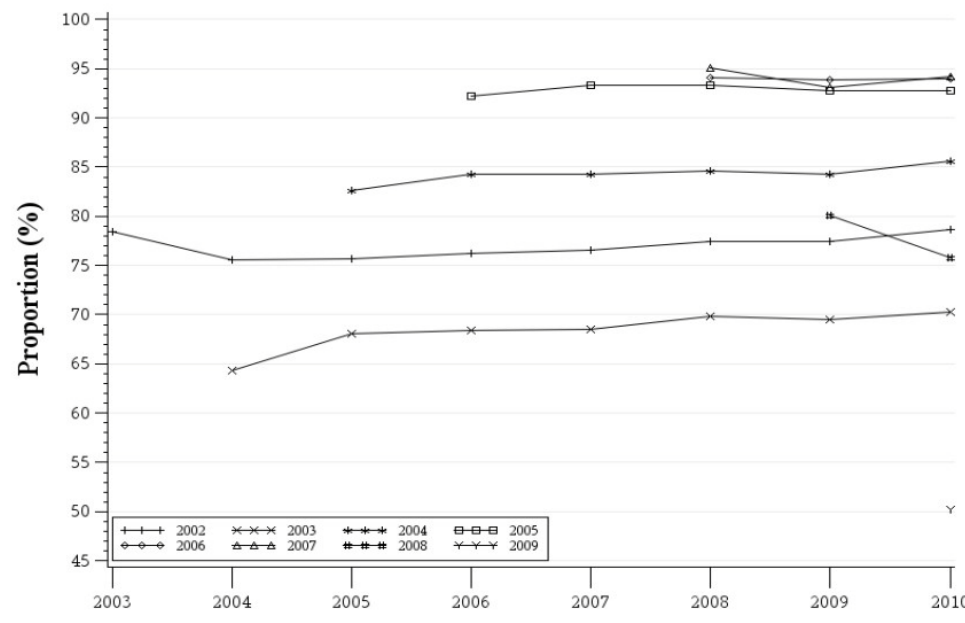

Echantillon : ensemble des contrats Madelin souscrits entre 2002 et 2009. Les parts investies en UC de l'année 2006 sont obtenues par interpolation au niveau contrat.

Notes : Les parts d’UC sont observées au 31 décembre. Elles sont datées en abscisse le 1er janvier de l'année suivante. 
Graphique 7. L’évolution des parts investies en unités de compte, conditionnellement à investir, décomposée par années d'ouverture du contrat

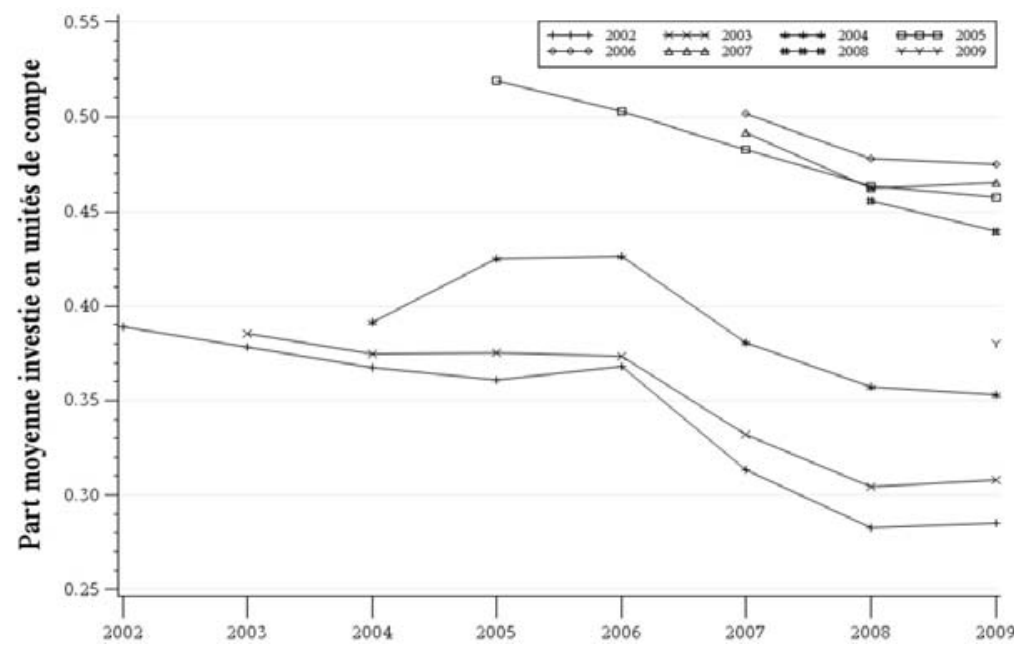

Echantillon : ensemble des contrats Madelin souscrits entre 2002 et 2009. Les parts investies en UC de l'année 2006 sont obtenues par interpolation au niveau contrat.

Notes : Les parts d’UC sont observées au 31 décembre. Elles sont datées en abscisse le 1er janvier de l'année suivante.

Graphique 8. Vue en coupe transversale des parts investies en unités de compte selon l’âge.

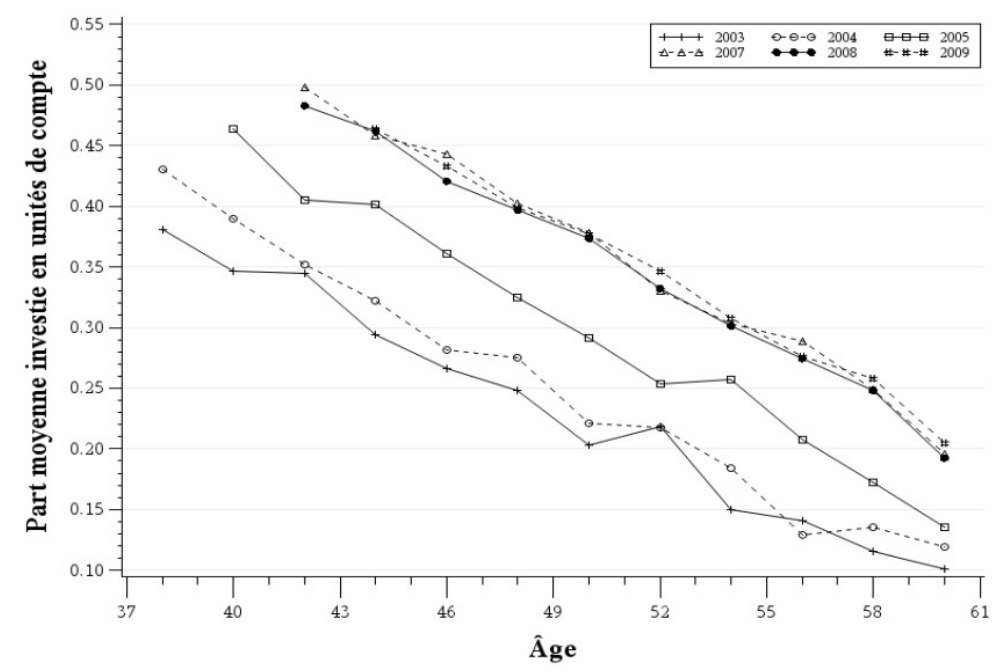

Echantillon : ensemble des contrats Madelin souscrits entre 2002 et 2009, exceptée l'année 2006. 
Graphique 9. Evolution des parts investies en unités de compte en fonction de l'âge, par dates de naissance.

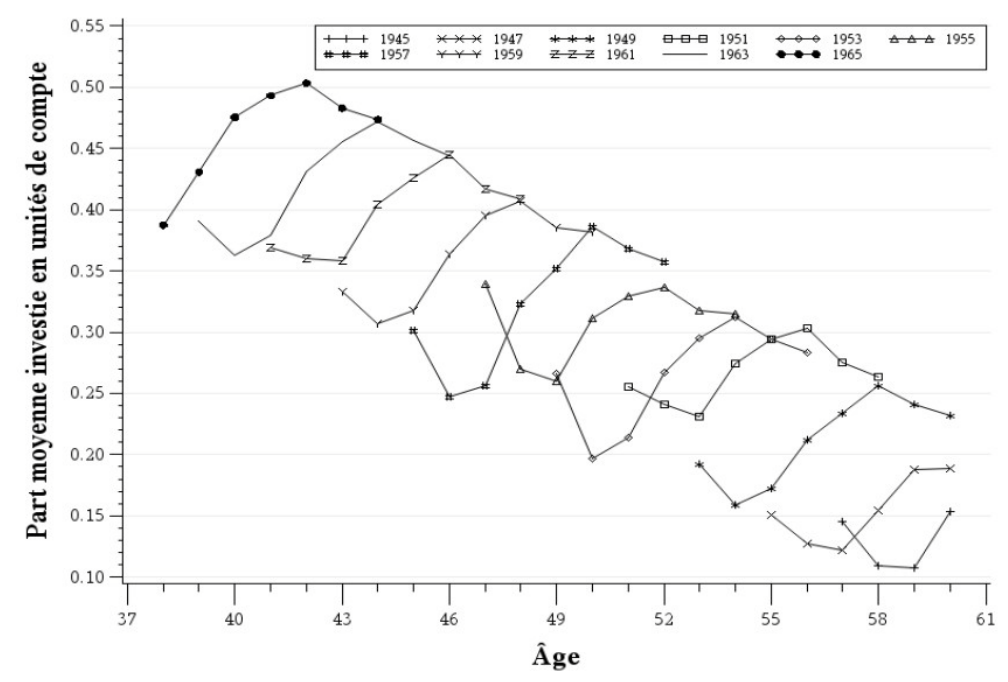

Echantillon : ensemble des contrats Madelin souscrits entre 2002 et 2009. Les parts investies en UC de l'année 2006 sont obtenues par interpolation au niveau contrat. Les épargnants nés avant 1945 sont regroupés avec ceux nés en 1945.

Note : Pour des raisons de clarté, seules les parts d’UC des épargnants nés une année impaire sont reproduites. Les parts d’UC des épargnants nés une année paire ont un profil similaire et s'intercalent entre les courbes des années impaires. 
Graphique 10. L'évolution des parts d'UC selon l'âge par cohortes de naissance et par dates de souscription

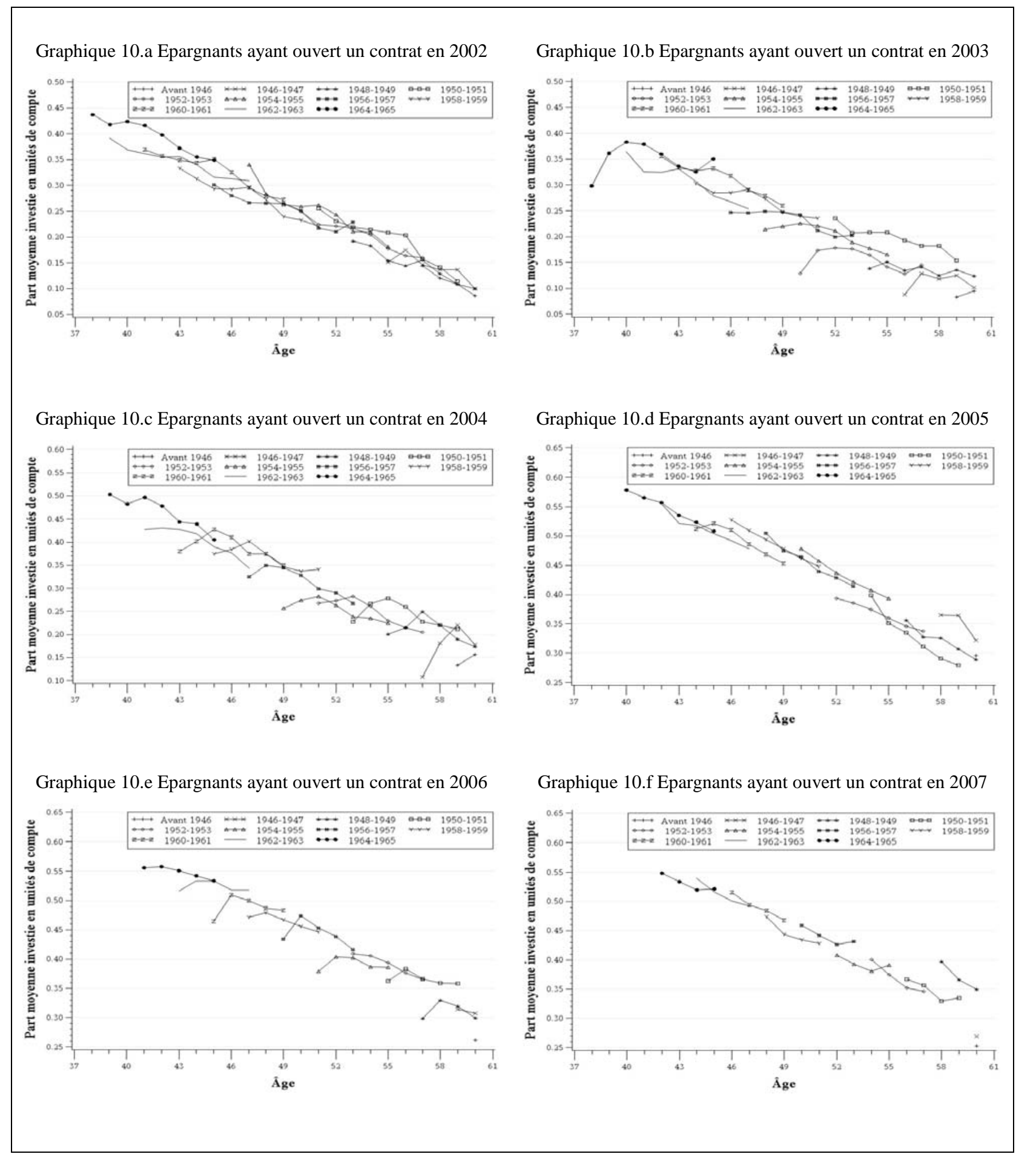

Echantillon : ensemble des contrats Madelin souscrits entre 2002 et 2009. Les parts d'UC de l'année 2006 sont obtenues par interpolation au niveau contrat.

Note : les graphiques des nouveaux souscripteurs de 2008 et 2009 sont omis, faute d'années suffisantes pour évaluer l'évolution de la part d’UC. 
Graphique 11. Estimation par régression Tobit des effets marginaux de l'âge sur la part d'unités de compte pour différents contrôles

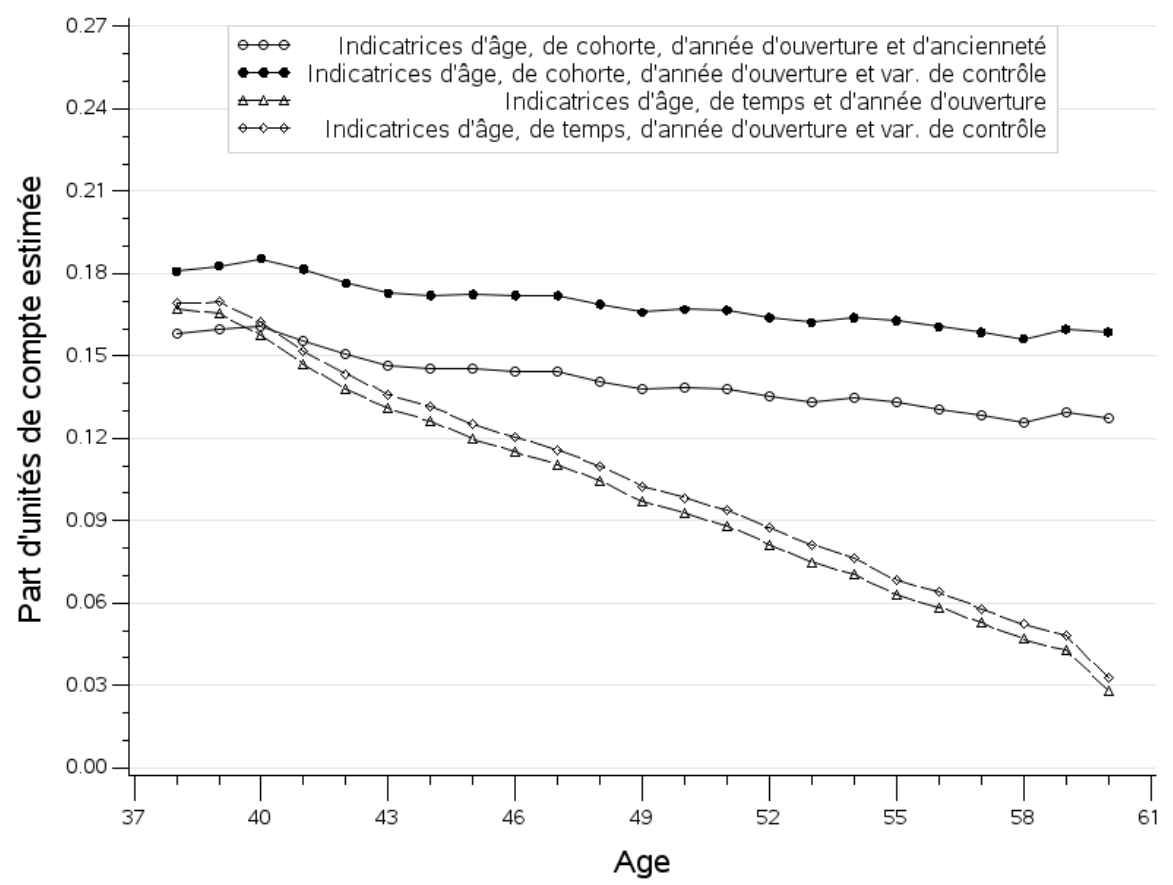

Echantillon : ensemble des contrats Madelin souscrits entre 2002 et 2009, exceptée l'année 2006.

Note : ce graphique indique de combien varie la part moyenne d'unités de compte quand l'âge augmente. Les effets marginaux sont estimés à l'aide d'un modèle de maximum de vraisemblance. Les épargnants âgés de plus de 60 ans sont regroupés avec les 60 ans. L’ancienneté est le nombre d'années écoulées entre l'année d'ouverture du contrat et celle du versement. Par variables de contrôles nous entendons le sexe du souscripteur, sa situation familiale, le nombre d'enfants, son département d'habitation, sa catégorie professionnelle, un indicateur de profil patrimonial et de revenu, le canal de distribution du contrat, le versement annualisé et le nombre annuel de versements. 
Graphique 12 Estimation des effets marginaux de l'âge sur la probabilité d'investir en unités de compte par régression d'un Probit pour différents contrôles

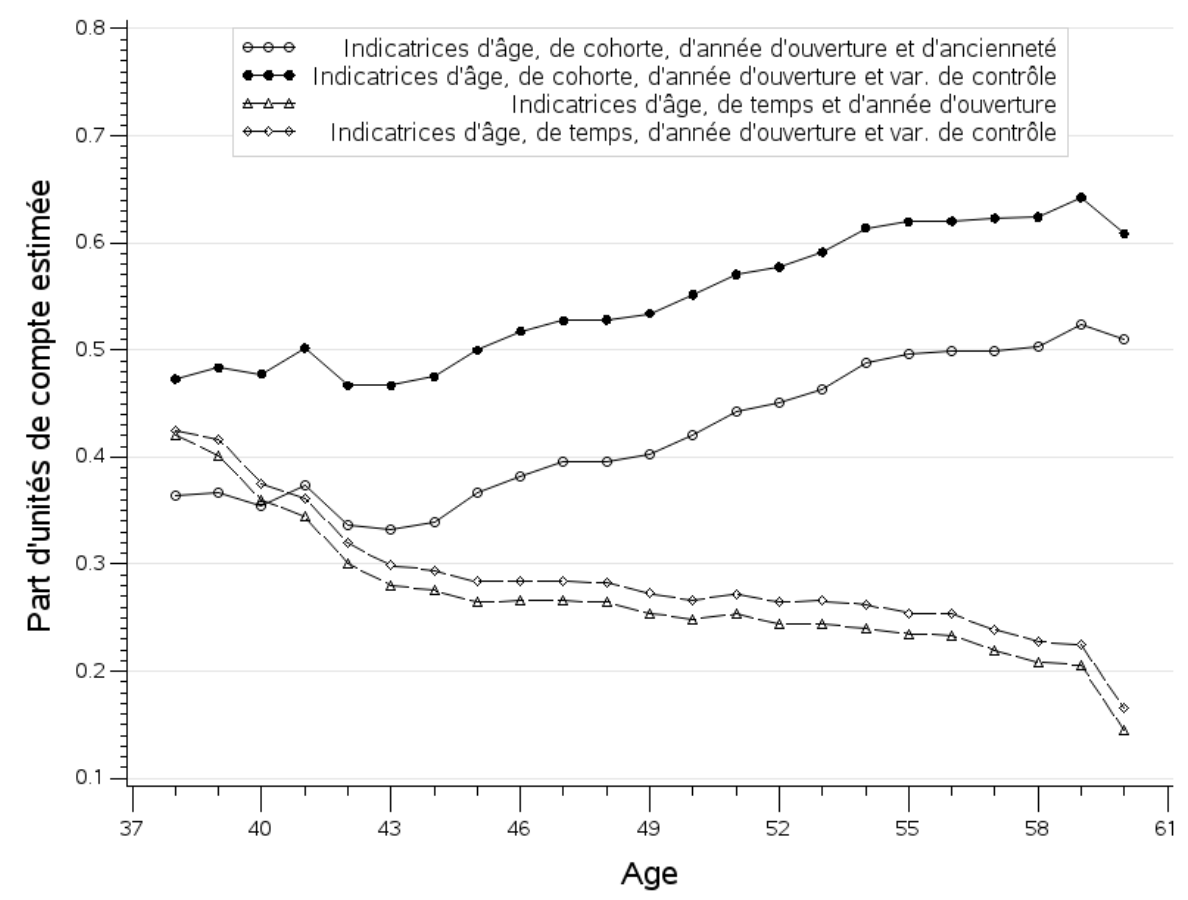

Echantillon : ensemble des contrats Madelin souscrits entre 2002 et 2009, exceptée l'année 2006.

Note : ce graphique présente l'évolution de la probabilité de détenir des unités de compte quand l'âge augmente. Les épargnants âgés de plus de 60 ans sont regroupés avec les 60 ans. L'ancienneté est le nombre d'années écoulées entre l'année d'ouverture du contrat et celle du versement. Les variables de contrôles regroupent le sexe du souscripteur, sa situation familiale, le nombre d'enfants, son département d'habitation, sa catégorie professionnelle, un indicateur de profil patrimonial et de revenu, le canal de distribution du contrat, le versement annualisé et le nombre annuel de versements. 
Graphique 13 Estimation par régression Tobit des effets marginaux de l’âge sur la part d'unités de compte, conditionnellement à investir, pour différents contrôles

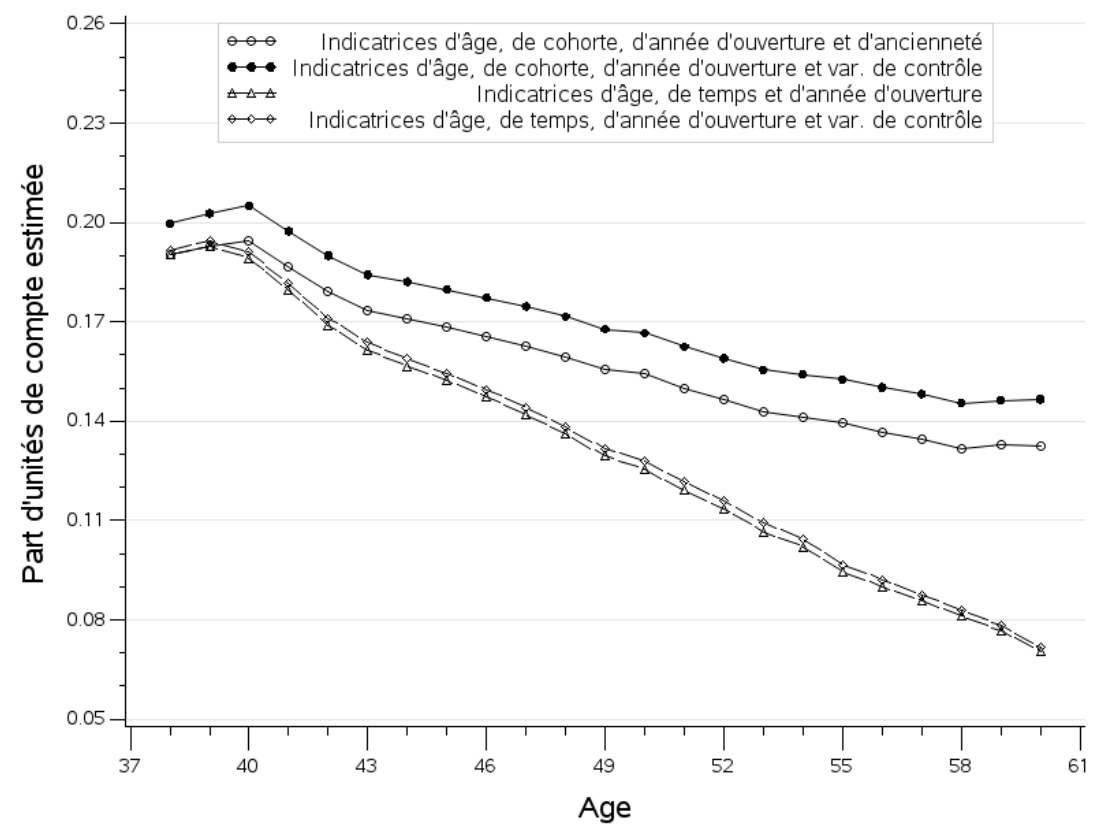

Echantillon : ensemble des contrats Madelin souscrits entre 2002 et 2009, exceptée l'année 2006.

Note : ce graphique indique de combien s'accroît la part d'unités de compte conditionnellement à investir quand l'âge augmente. Les effets marginaux sont estimés à l'aide d'un modèle de maximum de vraisemblance. Les épargnants âgés de 60 ans et plus sont regroupés avec les 60 ans. L'ancienneté est le nombre d'années écoulées entre l'année d'ouverture du contrat et celle du versement. Les variables de contrôles regroupent le sexe du souscripteur, sa situation familiale, le nombre d'enfants, son département d'habitation, sa catégorie professionnelle, un indicateur de profil patrimonial et de revenu, le canal de distribution du contrat, le versement annualisé et le nombre annuel de versements. 
Graphiques 14. Représentation graphique des effets marginaux de la date de naissance, de l'année de souscription et de l'année d’observation sur la probabilité de détenir des parts d’UC et sur la part investie en UC conditionnellement à investir

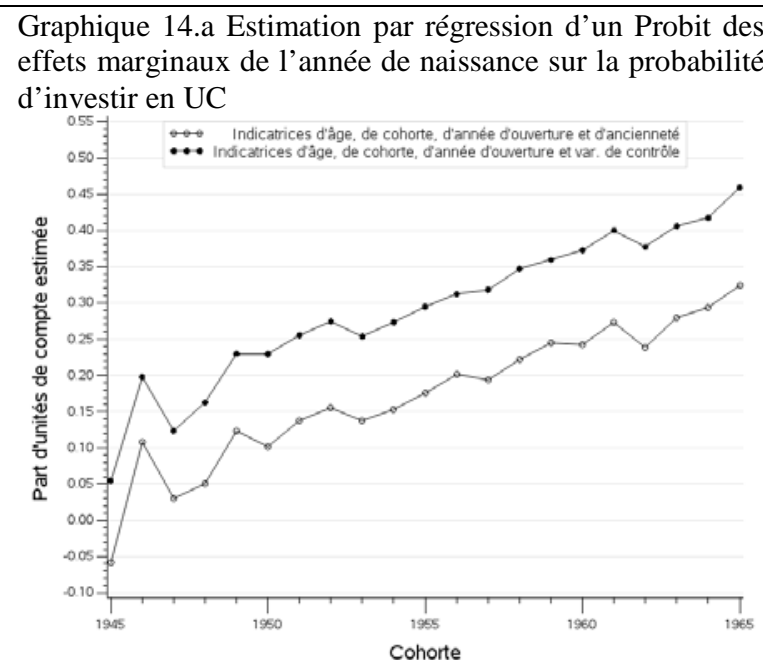

Graphique 14.a Estimation par régression d'un Probit des effets marginaux de l'année de souscription sur la probabilité d'investir en UC

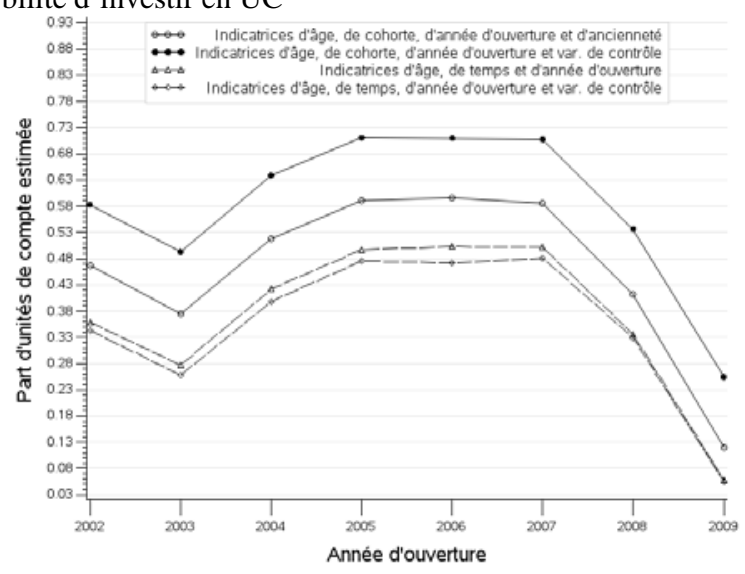

Graphique 14.e Estimation par régression d'un Probit des effets marginaux de l'année d'observation sur la probabilité d'investir en UC

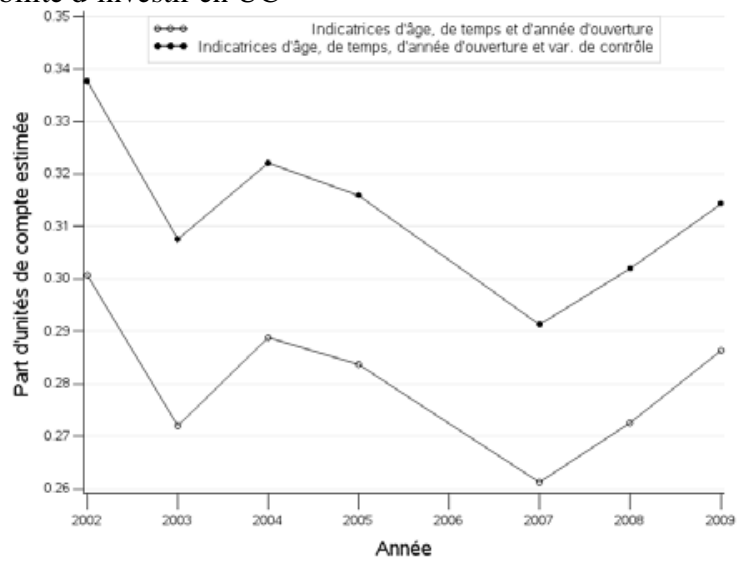

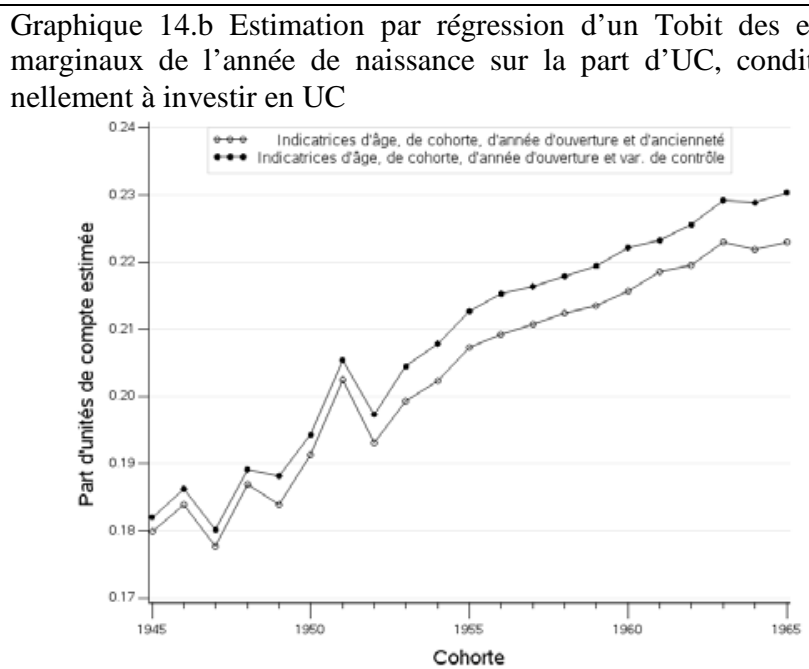

Graphique 14.b Estimation par régression d'un Tobit des effets marginaux de l'année de souscription sur la part d'UC, conditionnellement à investir en UC

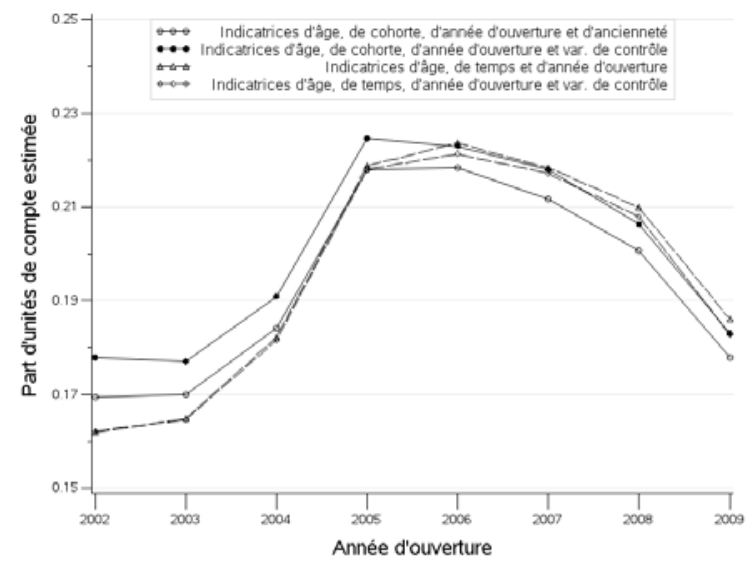

Graphique 14.b Estimation par régression d'un Tobit des effets marginaux de l'année d'observation sur la part d'UC, conditionnellement à investir en UC

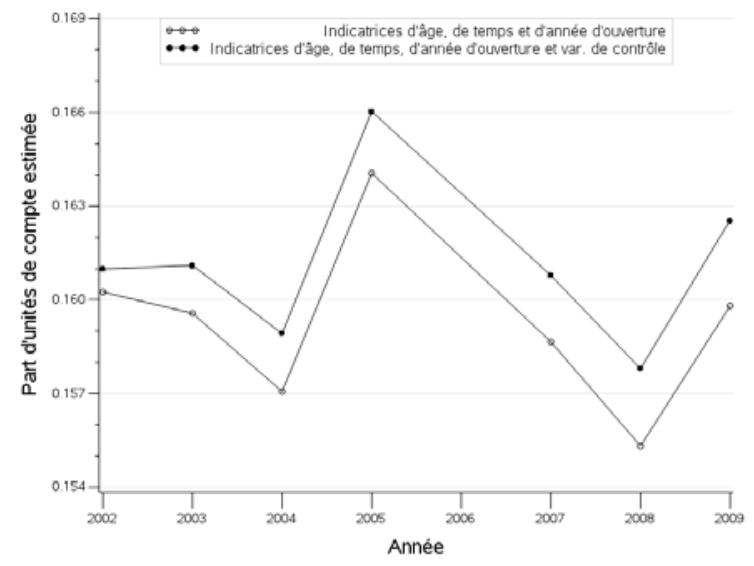

Echantillon : ensemble des contrats Madelin souscrits entre 2002 et 2009. Les parts d’UC de l'année 2006 sont obtenues par interpolation au niveau contrat. Note : Le tableau présente les effets marginaux sur la part investie en UC des indicatrices de cohorte ( $1^{\text {ère }}$ ligne), d'année d'ouverture ( $2^{\text {ème }}$ ligne) et d'année d'observation ( ${ }^{\text {ème }}$ ligne) dans les régressions Probit estimant la probabilité d'investir en UC (1 ${ }^{\text {ere }}$ colonne) et dans les régressions Tobit de la part d’UC conditionnellement à acheter des UC ( $2^{\text {nde }}$ colonne). 